\title{
FROM INTERSTELLAR DUST TO COMETS: A UNIFICATION OF OBSERVATIONAL CONSTRAINTS
}

\author{
J. Mayo Greenberg and J. I. Hage \\ University of Leiden \\ Received 1989 November 13; accepted 1990 March 20
}

\begin{abstract}
The interstellar dust model of comets is numerically worked out to satisfy simultaneously several basic constraints provided by observations of comet Halley, and to derive the porosity of coma dust. The observational constraints are (1) the strengths of the 3.4 and $9.7 \mu \mathrm{m}$ emission bands; (2) the shape of the $9.7 \mu \mathrm{m}$ band; (3) the relative amount of silicates to organic materials; (4) the mass distribution of the dust. The method used involves precise calculations of the temperatures and emission characteristics of porous aggregates of interstellar dust as a function of their mass, porosity, and distance to the Sun and the wavelength. The results indicate that coma dust has a porosity in the range $0.93<P<0.975$, i.e., a packing factor of 0.07 or less, consistent with independent observations of comet densities of $0.6 \mathrm{~g} \mathrm{~cm}^{-3}>\rho_{c}>0.26 \mathrm{~g} \mathrm{~cm}^{-3}$ and meteor densities of less than $0.2 \mathrm{~g} \mathrm{~cm}^{-3}$.
\end{abstract}

Subject headings: comets - infrared: spectra — interstellar: grains — radiative transfer

\section{INTRODUCTION}

There have been many suggestions to explain how comets were born in the pre-solar system nebula. Although it is generally recognized that comets are the most primitive bodies in the solar system, the question is, are they made of unmodified protosolar nebula interstellar dust, or has this material been (completely or partially) evaporated before becoming a part of comets? The basic idea of the model of comets by Greenberg $(1977,1982,1985)$ is that in the regions of the protosolar nebula where comet formation took place, the temperature was so low that the dust particles were completely preserved during aggregation into a comet. There are several bases for substantiation of this model. First, at least one theoretical prediction states that the temperature between Uranus and Neptune, where many believe comets formed, was $25 \mathrm{~K}<T<67 \mathrm{~K}$ (Ruzmaikina and Maeva 1988). This temperature range is thought to be low enough to keep all dust components from evaporation. A second justification is the observation of $S_{2}$ as a parent molecule in comet IRAS-ArakiAlcock (A'Hearn, Feldman, and Schleicher 1983). This molecule was shown to be created in interstellar space by ultraviolet processing of icy mantles, and its presence suggests that the temperature of comet formation was well below that of $\mathrm{H}_{2} \mathrm{O}$ evaporation (Grim and Greenberg 1987a). A third justification is that the fraction of $\mathrm{CH}_{4}$ observed in comets Halley and Wilson is consistent with the chemistry of photoprocessed interstellar dust but "not with either equilibrium or disequilibrium condensation sequences in the (gaseous) solar nebula" (Larson et al. 1989). Finally, the interstellar dust model of comets has made it possible to predict some of the "surprising" comet Halley observations (Greenberg 1986a): the very low albedo of comet Halley, the presence of particles with masses as low as $10^{-17} \mathrm{~g}$ and the large organic component of coma dust.

In this paper we show that the interstellar dust model of comets can be numerically developed to satisfy, both simultaneously and quantitatively, the following observational constraints: (1) the observed strengths of the 3.4 and $9.7 \mu \mathrm{m}$ emission bands of comet Halley; (2) the shape of the $9.7 \mu \mathrm{m}$ band of comet Halley; (3) the relative amounts of silicates to organic materials measured in situ; (4) the mass distribution of the dust, which was also measured in situ. Furthermore, we show that a high coma dust porosity, as predicted by the comet model, is consistent with the above constraints. An earlier attempt was made to do this, using the size distribution given at that time by McDonnell et al. (1987). It was readily demonstrated by relatively simple methods that even with extremely high porosities it was impossible to reproduce the observed ratio of the 9.7 and $3.4 \mu \mathrm{m}$ emission bands unless the number of particles with $M<2 \times 10^{-9} \mathrm{~g}$ was between one and two orders of magnitude higher than allowed by the given size distribution (Greenberg, Zhao, and Hage 1989). Since then, McDonnell et al. have revised the small size coma dust distribution upward. The present paper uses this updated Giotto size distribution (McDonnell et al. 1989) and those obtained on the Vega probes (Mazets et al. 1987). Furthermore, rigorous calculations of the temperatures and spectral properties of porous coma dust particles are performed.

Section II provides a brief review of the interstellar dust model of comets and coma dust. In $\S$ III $a$ formulae are derived which show the relation between the observed size spectra in the coma and observations of the thermal emission. Section III $b$ lists our choice of observational results. In $\S \S$ IV and V we derive the emission properties and temperatures of porous coma dust particles as a function of their size, porosity, and composition. In $\S$ VI the results of $\S \S$ III-V are used to fit observational constraints. Section VII contains a discussion, and $\S$ VIII our conclusions.

\section{THE INTERSTELLAR DUST MODEL OF COMETS}

We briefly review the model of interstellar dust used here and derive the predicted properties of the comet nucleus and the coma dust according to that model.

\section{a) Interstellar Dust in the Protosolar Nebula}

Protosolar nebula dust, out of which comets are formed, is a developed stage of diffuse cloud interstellar dust (Greenberg 1985). The diffuse cloud interstellar dust consists of three major 
populations of particles as described by Greenberg (1985). The major population, in terms of mass, consists of elongated particles with a silicate core and an "organic refractory" mantle, a few tenths of a micron in size (i.e., "core-mantle particles"; see Fig. 1a). The organic refractory material originally started out as ices of simple chemical compounds, but has undergone up to billions of years of ultraviolet photoprocessing in interstellar space. The photoprocessing has changed the ice mixture into a carbon-rich and oxygen-poor refractory material containing many different organic molecules. The second population contains very small carbonaceous particles with radii smaller than $10^{-2} \mu \mathrm{m}$. Equally small silicate particles were originally proposed for the third population by Greenberg, but recent developments indicate that this population may consist of polycyclic aromatic hydrocarbon (PAH) molecules instead (see, e.g., Leger, d'Hendecourt, and Boccara 1987).

In the presolar molecular cloud the dust consists of the coremantle particles with an additional outer mantle of volatile ices dominated by $\mathrm{H}_{2} \mathrm{O}$ (see Fig. $1 b$ ). The expected mass fractions
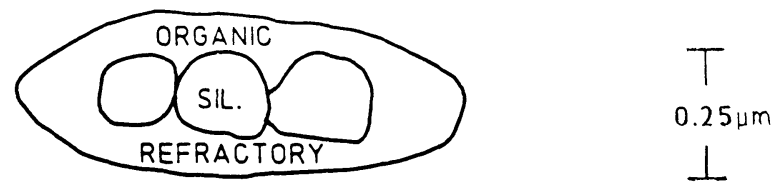

DIFFUSE CLOUD GRAIN.
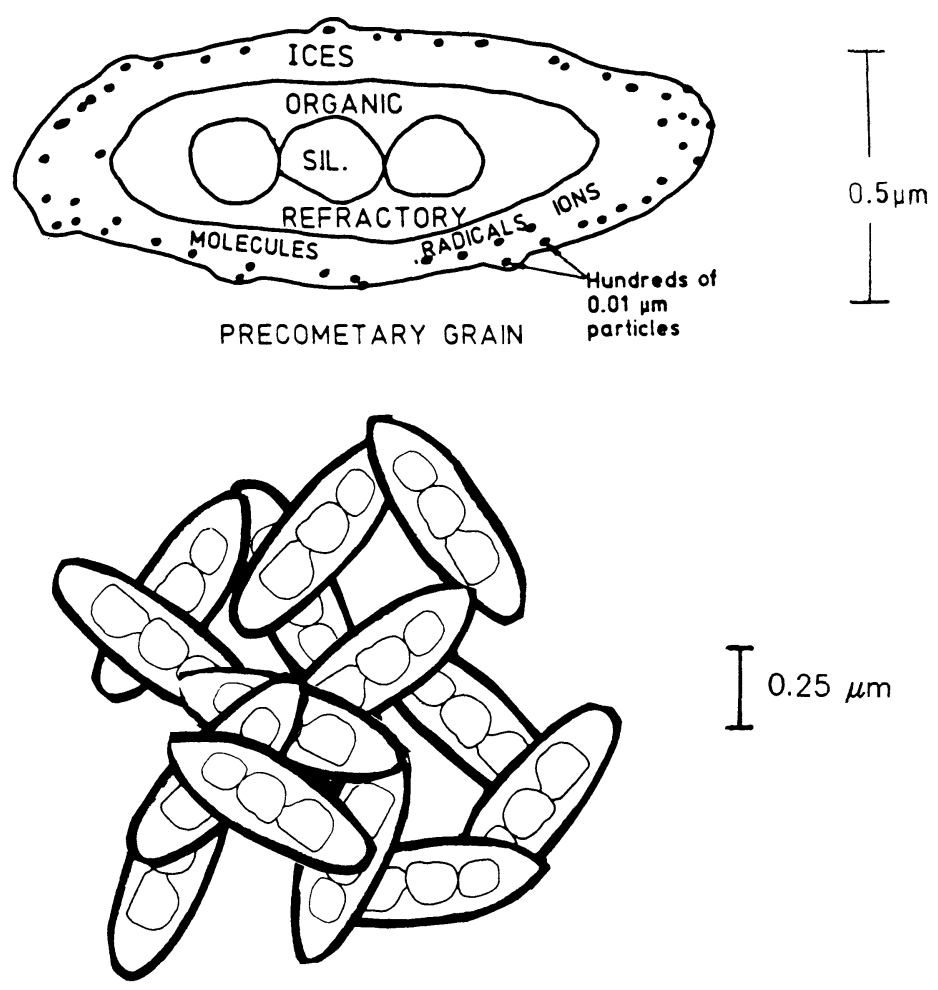

COMA GRAIN

Fig. 1.-Top: Schematic of an interstellar dust grain which contains a core of silicates and a mantle of organic refractory material. Middle: An interstellar grain as it would appear in the protosolar dust cloud after accretion of gases on its surface. These grains make up a comet. Bottom: Schematic of a coma grain as it would appear according to the interstellar dust model. It would have a porosity of between about 0.93 and 0.98 . of the three components of this dust are $0.20,0.19$, and 0.55 , for the silicate (subscript "sil" in what follows), organic refractory ("or"), and volatile material ("ice"), respectively. These proportions are based on the model of interstellar dust (Greenberg 1988) extrapolated to the protosolar nebular cloud stage and constrained by solar abundance. In a very dense region all the smaller particles could be embedded in the icy mantles. The mass fraction of carbonaceous particles (subscript $c$ ) is about 0.06 .

\section{b) The Comet Nucleus and Coma Dust}

Regarding the formation of comets, it has been proposed by Greenberg that, initially, aggregates of the protosolar nebula particles are made. Their tangled structure, like a bird's nest (Greenberg and Gustafson 1981), is suggested as a possible way to provide them with rigidity. This structure may also be conjectured to be the result of random aggregation modified by slippage after particle collision at speeds of about $0.1 \mathrm{~km} \mathrm{~s}^{-1}$. The aggregates, in turn, coalesce into larger bodies and ultimately comets. When a comet comes close to the Sun, individual aggregates of various sizes are lifted from the comet and are exposed to the solar radiation. The ice mantles evaporate, and the small carbonaceous particles are lost from the aggregates as well. Some of the organic material may also evaporate. Hereafter the aggregates consist of core-mantle particles composed only of organic refractory material and interstellar silicates as illustrated schematically in Figure 1c. It is assumed that, under these conditions, this type of porous particle constitutes the coma dust in all but the innermost part of the coma. This assumption immediately implies that the 3.4 and $9.7 \mu \mathrm{m}$ spectral features characteristic of interstellar dust should, under appropriate conditions, show up in the infrared coma emission of a comet.

We now define the porosity, $P$, of an aggregate of interstellar dust as

$$
P=1-\frac{V_{\text {solid }}}{V}
$$

where $V_{\text {solid }}$ is the volume of the solid material inside the aggregate and $V$ is the total volume of the aggregate, its volume being defined by an appropriate boundary. We define this boundary as the surface of the smallest convex volume which contains all the solid material of the aggregate (see Hage and Greenberg $1990 a$ for an example). We have $0 \leq P<1$, where $P=0$ corresponds to a solid aggregate and $P \approx 1$ corresponds to a cloud of independent interstellar dust particles. Note that a porosity $P$ corresponds to a packing factor of $p=1-P$, and that if the porosity is high, a small change in the porosity corresponds to a large relative change in the packing factor. The interstellar dust model of comets predicts that the porosity of the comet could be as high as $P_{\text {nuc }}=0.83$, i.e., $83 \%$ of the total volume of a comet could be taken up by empty space (a packing factor of $p=0.17$ ). The predicted porosity of the coma dust could be as high as $P_{\text {dust }}=0.975$. These predictions are clarified in the remainder of this section.

If the aggregation into a comet were to lead to a fully compacted structure (i.e., $P=0$ ), the nucleus density would be

$$
\rho_{\text {nuc }}=\frac{M_{\text {sil }}+M_{\text {or }}+M_{c}+M_{\text {ice }}}{V_{\text {sil }}+V_{\text {or }}+V_{c}+V_{\text {ice }}},
$$

where $M$ and $V$ refer to the mass and volume fractions of the various materials in the protosolar nebula dust. Using the 
mass fractions of the protosolar nebula dust given in $\S$ II $a$ and densities of $\rho_{\text {sil }}=3.5, \rho_{\text {or }}=1.8, \rho_{\text {ice }}=1.2$, and $\rho_{c}=2$, in units of grams per cubic centimeter, one finds $\rho_{\text {nuc }}=1.54 \mathrm{~g} \mathrm{~cm}^{-3}$. This is about $15 \%$ higher than previously derived (Greenberg $1986 \mathrm{~b}$ ), because of the present assumption of higher material densities. The present result assumes that the condensable elements in the comet have (proto)solar abundance. The density and porosity of the coma dust may now be derived by assuming that the only difference between comet material and coma dust is that the volume filled originally by the ices and small carbonaceous particles in the nucleus material is taken up by an equal volume of vacuum inside the coma dust. This corresponds to taking $M_{c}=M_{\text {ice }}=0$ in equation (2), resulting in $\rho_{\text {dust }}=0.6 \mathrm{~g} \mathrm{~cm}^{-3}$. The porosity of the coma dust derived in this way would be

$$
P_{\text {dust }}=1-\frac{V_{\text {sil }}+V_{\text {or }}}{V_{\text {sil }}+V_{\text {or }}+V_{c}+V_{\text {ice }}}=0.75 .
$$

At this point we note that the mean density of meteors, some of which are clearly of cometary origin, is generally much less than $0.6 \mathrm{~g} \mathrm{~cm}^{-3}$. According to Verniani (1969, 1973), those meteors whose mean aphelion distance is highest and which are therefore least affected by the Sun have the lowest densities of about $0.2 \mathrm{~g} \mathrm{~cm}^{-3}$. The interstellar dust model of comets assumes that the density of coma dust equals, or is less than, that of these meteors, and this implies that the coma dust must have a higher porosity than derived above. The implied minimum density change by a factor of 3 now gives

$$
P_{\text {dust }}=1-\frac{V_{\text {sil }}+V_{\text {or }}}{3\left(V_{\text {sil }}+V_{\text {or }}+V_{c}+V_{\text {ice }}\right)}=0.92 \text {. }
$$

Consequently, by reconstitution of the ices and carbonaceous particles into the coma dust, the porosity and density of the comet itself may be derived:

$$
\begin{gathered}
P_{\text {nuc }}=1-\frac{V_{\text {sil }}+V_{\text {or }}+V_{\text {ice }}+V_{c}}{3\left(V_{\text {sil }}+V_{\text {or }}+V_{c}+V_{\text {ice }}\right)}=0.67, \\
\rho_{\text {nuc }}=\frac{M_{\text {sil }}+M_{\text {or }}+M_{c}+M_{\text {ice }}}{3\left(V_{\text {sil }}+V_{\text {or }}+V_{c}+V_{\text {ice }}\right)}=0.51 .
\end{gathered}
$$

Alternatively, one may assume a meteor density of $0.1 \mathrm{~g} \mathrm{~cm}^{-3}$ as given by Olsson-Steel (1989), which would give $\boldsymbol{P}_{\text {dust }}=0.96$, $P_{\text {nuc }}=0.83, \rho_{\text {nuc }}=0.26$. What a porosity of 0.8 implies for the morphology of a particle is shown in Figure $2 a$ (Plate 1) by a model consisting of a hundred particles. Another possibility is to take the comet density of $0.6 \mathrm{~g} \mathrm{~cm}^{-3}$ (as derived by Sagdeev, Elyasberg, and Moroz 1988) as a starting point and use the same volume and mass fractions as above, to derive the comet and coma dust porosities. These then turn out to be $P_{\text {dust }}=$ 0.91 and $P_{\text {nuc }}=0.61$. Finally, one could start with a comet density of $0.25 \mathrm{~g} \mathrm{~cm}^{-3}$, derived from the splitting of the comet Brooks 2 when it passed by Jupiter (Sekanina and Yeomans 1985). This would give the same results as using the meteor density of Olsson-Steel (1989). A summary of these results is contained in Table 1, where we have included the possibility for partial evaporation of the organic refractory component which actually is expected at high temperatures. Note that under the present assumptions, the lowest possible coma dust porosity (which corresponds to a fully compact comet nucleus) is $P=0.75$, or $P=0.83$ if half of the organics have evaporated.

\begin{tabular}{|c|c|c|c|c|c|}
\hline $\begin{array}{c}\rho_{\text {nuc }} \\
\left(\mathrm{g} \mathrm{cm}^{-3}\right)\end{array}$ & $P_{\text {nuc }}$ & $P_{\text {dust }}^{1: 1}$ & $P_{\text {dust }}^{2: 1}$ & $\begin{array}{c}\rho_{\text {dust }}^{1: 1} \\
\left(\mathrm{~g} \mathrm{~cm}^{-3}\right)\end{array}$ & $\begin{array}{c}\rho_{\text {dust }}^{2: 1} \\
\left(\mathrm{~g} \mathrm{~cm}^{-3}\right)\end{array}$ \\
\hline $1.54 \ldots \ldots \ldots$ & 0. & 0.75 & 0.83 & 0.60 & 0.45 \\
\hline $0.60 \ldots \ldots \ldots$ & 0.61 & 0.906 & 0.933 & 0.23 & 0.18 \\
\hline $0.51 \ldots \ldots \ldots$ & 0.67 & 0.92 & 0.94 & 0.20 & 0.16 \\
\hline $0.26 \ldots \ldots \ldots$ & 0.83 & 0.96 & 0.975 & 0.10 & 0.08 \\
\hline
\end{tabular}

TABLE 1

Comet Nucleus Densities and Porosities, with CoRresponding Coma Dust Densities and Porosities ${ }^{a}$

a The superscripts denote $M_{\text {sil }}: M_{\text {or }}$ in the dust. The case $1: 1$ is the original cometary ratio according to the model, and the case 2:1 corresponds to the situation where half of the organic refractory has evaporated.

\section{METHOD}

\section{a) Theory}

For the purpose of our initial discussion, we consider the coma of comet Halley to be a spherically symmetric cloud of gas and dust, in which the particle density decreases as the inverse square of the distance to the comet nucleus. We shall assume that the outward flow of dust particles is directed radially from the nucleus and that the particle mass spectrum does not depend on the distance to the nucleus in the inner coma. Let the flux (per unit wavelength) observed at Earth from the coma, seen within an aperture defined by a radius $R_{a}$ at the comet, be denoted by $F(\lambda)$. Then the total power, $W^{o}$, emitted in all directions from the central spherical region within the aperture is

$$
W^{o}=\frac{2}{3} 4 \pi \Delta^{2} F(\lambda),
$$

where $\Delta$ is the distance from the Earth to the comet at the time of observation. The factor $\frac{2}{3}$ takes into account to a good approximation that the emission as seen from Earth comes from a cylindrical volume rather than from a sphere.

We define the mass absorption coefficient of a particle, $\kappa(\lambda)$, as

$$
\kappa(\lambda)=\frac{C_{\mathrm{abs}}(\lambda)}{M},
$$

where $C_{\mathrm{abs}}$ is the cross section for absorption of the particle and $M$ is its mass. The power emitted by a spherical particle at a temperature $T$ is then given by the product $4 \pi M \kappa(\lambda) B(\lambda, T)$, where $B(\lambda, T)$ is the Planck function. In terms of the particles in the observed central spherical part of the coma, assuming that particles with the same mass are identical, the total emitted power per unit wavelength, $W^{e}$, is

$$
W^{e}=4 \pi\left(4 \pi R_{a}^{3}\right) \int_{0}^{\infty} \kappa(\lambda) B(\lambda, T) M n(M) d M,
$$

where $n(M) d M$ is the number of particles of mass $M$ per unit volume at a distance $R_{a}$ from the comet, in the mass range $d M$. A theoretical fit of the observed spectrum must therefore satisfy

$$
4 \pi R_{a}^{3} \int_{0}^{\infty} \kappa(\lambda) B(\lambda, T) M n(M) d M=\frac{2}{3} \Delta^{2} F(\lambda)
$$

for all wavelengths where the thermal emission dominates the reflected solar radiation.

Basic to this work is the consideration of the emission bands at 3.4 and $9.7 \mu \mathrm{m}$, which are clearly distinguishable from the continuum radiation from the coma. For either of these bands 
we define the excess emission above the continuum, $F^{\mathrm{ex}}$, as

$$
F^{\mathrm{ex}}(\lambda)=F(\lambda)-\left[F\left(\lambda_{1}\right)+\frac{F\left(\lambda_{2}\right)-F\left(\lambda_{1}\right)}{\lambda_{2}-\lambda_{1}}\left(\lambda-\lambda_{1}\right)\right] .
$$

Here $\lambda_{1}$ and $\lambda_{2}$ are the lower and upper wavelengths of the bands, respectively. If we also define the effective mass absorption coefficient for a particle and for these bands, $\kappa^{\text {eff }}$, as

$$
\begin{aligned}
\kappa^{\text {eff }}(\lambda)=\frac{1}{M} & \left\{C_{\text {abs }}(\lambda)\right. \\
- & {\left.\left[C_{\mathrm{abs}}\left(\lambda_{1}\right)+\frac{C_{\mathrm{abs}}\left(\lambda_{2}\right)-C_{\mathrm{abs}}\left(\lambda_{1}\right)}{\lambda_{2}-\lambda_{1}}\left(\lambda-\lambda_{1}\right)\right]\right\}, }
\end{aligned}
$$

then, if there is a well-defined emission band between $\lambda_{1}$ and $\lambda_{2}$, equation (10) becomes, to a good approximation,

$$
4 \pi R_{a}^{3} \int_{0}^{\infty} \kappa^{\mathrm{eff}}\left(\lambda_{p}\right) B\left(\lambda_{p}, T\right) M n(M) d M=\frac{2}{3} \Delta^{2} F^{\mathrm{ex}}\left(\lambda_{p}\right)=\frac{W^{\mathrm{ex}}}{4 \pi}
$$

for $\lambda_{1}<\lambda_{p}<\lambda_{2}$. Here $\lambda_{p}$ denotes the wavelength of the band peak. This approximation breaks down in case the Planck function has a sharper peak than $\kappa^{\text {eff }}$, which is, however, not the case we are interested in. Equation (13) shows how the relation between two observed quantities, $F^{\mathrm{ex}}(\lambda)$ and $n(M)$, depends on the properties of the emitting dust, through the quantities $\kappa^{\text {eff }}$ and $T$. It is very important to note here that the consideration of the excess emission above the continuum, rather than the continuum itself, facilitates a model fit to the observations and alleviates the need to integrate over the largest particles in the size distribution (for which in situ measurements are uncertain). This is because $\kappa^{\text {eff }}(\lambda)$ becomes very small for large particles (as will be shown in $\S$ IV), so that their contribution to the excess emission is negligible.

In this paper we shall assume that the coma dust has a certain porosity which is unknown, and which must therefore be taken as a free parameter in the calculations of the coma dust emission. The goal of this work is to calculate the lefthand side of equation (13) as a function of porosity of the coma dust and then, by equating left- and right-hand sides, use equation (13) to determine the porosity of the coma dust. In particular, we shall satisfy equation (13) for the 3.4 and $9.7 \mu \mathrm{m}$ bands. We shall calculate the quantities $\kappa^{\text {eff }}$ and $T$ of the coma dust particles exactly, based on the composition and structure of coma dust as derived from the interstellar dust model. The dependence of $\kappa^{\text {eff }}$ and $T$ on particle size (or mass $M$ ), particle porosity $(P)$, wavelength $(\lambda)$, and distance to the Sun $(r)$ will be taken fully into account. Furthermore, we use for $n(M)$ the dust mass spectra as measured in situ by the spacecraft missions to comet Halley, thus implicitly satisfying this observational constraint. The radius of the silicate cores in the individual interstellar core-mantle particles is fixed at $0.075 \mu \mathrm{m}$. Last, the relative size of the core and mantle are taken so that the mass ratio of the silicates to organic material is either $1: 1,2: 1,5: 1$, or $1: 0$. The ratio $1: 1$ corresponds closely to the initial average ratio in the nucleus (according to the interstellar dust model), and the ratio 2:1 was measured in the coma (Kissel and Krueger 1987). The other ratios are included to show the effect of variation of this parameter. In $\S \S \mathrm{IV}$ and $\mathrm{V}$ the calculation of $\kappa^{\text {eff }}(\lambda, P, M)$ and $T(P, M, r)$ are discussed in more detail.

\section{b) Observations}

Because of variability in the dust emission, it is necessary to choose observations of $n(M)$ and $F^{\mathbf{e x}}(\lambda)$ as close together in time as possible. The Vega 1, Vega 2, and Giotto closest approaches occurred on March 6, 9, and 14, respectively. We have therefore chosen to use the observations by Hanner et al. (1987) on March 6.85, 12.8, and 13.75. These observations determine the values of $\lambda_{1}=7.8 \mu \mathrm{m}$ and $\lambda_{2}=12.5 \mu \mathrm{m}$ to be used in equation (12). The values we obtained for $F^{\mathrm{ex}}(9.7)$ on these dates are $6.6,5.4$, and 3.4 in units of $10^{-16} \mathrm{~W} \mathrm{~cm}$ $\mu \mathrm{m}^{-1}$. As far as we know, no observations of the $3.4 \mu \mathrm{m}$ band exist on these days. Therefore we are required to take observations at a later date (March 28) to obtain the ratio of the $9.7 \mu \mathrm{m}$ excess to the $3.4 \mu \mathrm{m}$ excess, and we use this ratio to estimate the value of $F^{\mathrm{ex}}(3.4)$ on March $6.85,12.8$, and 13.75. The silicate emission deduced from observations on March 28.6 by Gehrz and Ney (1986) is $F^{\text {ex }}(9.7)=1.2 \times 10^{-16} \mathrm{~W} \mathrm{~cm}^{-2} \mu \mathrm{m}^{-1}$, using a smaller aperture than Hanner et al. From Danks et al. (1987) we obtained $F^{\mathrm{ex}}(3.4)=2.6 \times 10^{-17} \mathrm{~W} \mathrm{~cm}^{-2} \mu \mathrm{m}^{-1}$. After normalizing these values to a single aperture, we obtain a ratio of about 10. However, we note that the measurements by Gehrz and Ney did not include the $7.8 \mu \mathrm{m}$ filter, so that we used $\lambda_{1}=8.5 \mu \mathrm{m}$ in equation (12) instead, thereby underestimating the strength of the $9.7 \mu \mathrm{m}$ band. On the other hand, because of the greater solar distance on March 28, we may be overestimating the ratio $F^{\mathbf{e x}}(9.7) / F^{\mathbf{e x}}(3.4)$ for the earlier dates. Consequently we shall take as a reasonable estimate $F^{\text {ex }}(9.7) /$ $F^{\text {ex }}(3.4)=10$ on March $6.85,12.8$, and 13.75.

The quantity $n(M)$, the differential number density of the coma dust particles, was obtained from the cumulative particle fluxes measured by the spacecraft. To derive $n(M)$ for the Giotto passage on March 14, we have used the curve labeled "coma," presented in Figure 1 of McDonnell et al. (1989). This curve gives the measured cumulative flux up to a mass of about $0.1 \mathrm{~g}$. This curve was matched analytically to a good accuracy with piecewise linear functions. The differential particle flux was obtained by differentiation of the analytical expression. Conversion from flux to density was accomplished by division by the spacecraft velocity relative to the comet nucleus. We note that because of a recalibration of the instruments, the $n(M)$ for $10^{-15} \mathrm{~g}<M<10^{-10} \mathrm{~g}$ deduced from this are a factor of up to 25 times larger than the values that would be deduced from the results as presented in McDonnell et al. (1987). We obtained $n(M)$ for the Vega missions, in a similar way, from Mazets et al. (1987) (their Figs. 5 and 8, top curves). These curves give the cumulative flux up to a mass of about $10^{-6} \mathrm{~g}$. We have extrapolated these fluxes, using the slope at this mass, to a mass of $0.1 \mathrm{~g}$. We note that the Vega and Giotto differential particle mass distributions are similar in shape and amount up to masses of about $10^{-10} \mathrm{~g}$, but that significant differences of up to two orders of magnitude occur in the differential number density in the mass range $10^{-10}$ to $10^{-4} \mathrm{~g}$. In Figures $3 a$ and $3 b$ we present the various size distributions obtained by the three spacecraft. In addition, we must take into account the spatial inhomogeneity of the coma. First, all three of these size spectra have been measured in the sunward hemisphere of the coma, where the particle densities are higher than in the other hemisphere. The ratio of Giotto post- and preencounter count rates indicate a density difference of a factor of 3 between the two hemispheres. The theoretical amount of emission as given by equation (13) must be multiplied by $\frac{2}{3}$ to take this into account. Second, whereas the Giotto spectrum we have used is an average over a path length of about $4100 \mathrm{~km}$ (McDonnell et al. 1989), the Vega data we have used are influenced more by the jetlike structure of the inner coma. Judging from Figure 10 of Mazets et al. (1987), it appears that the flux curves at the point of closest approach for the Vega spacecraft 


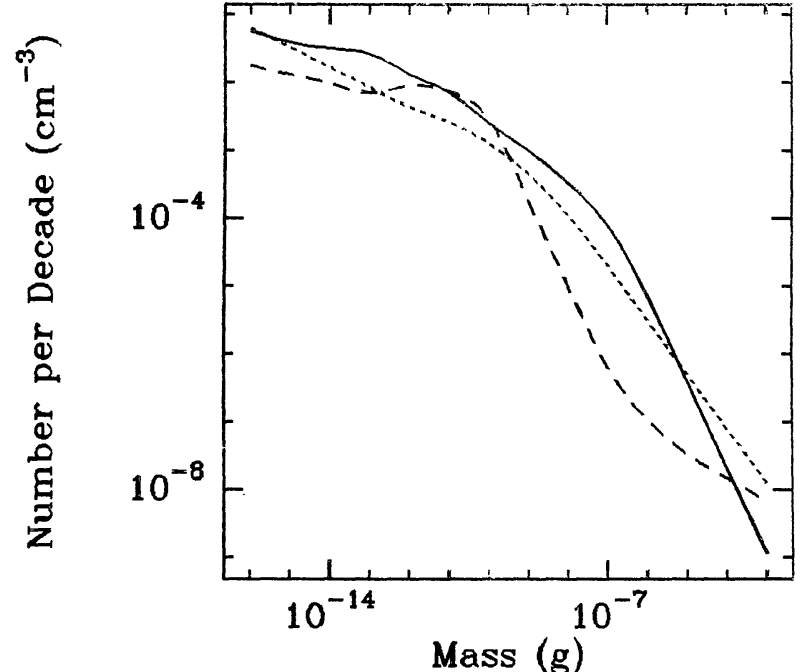

FIG. $3 a$

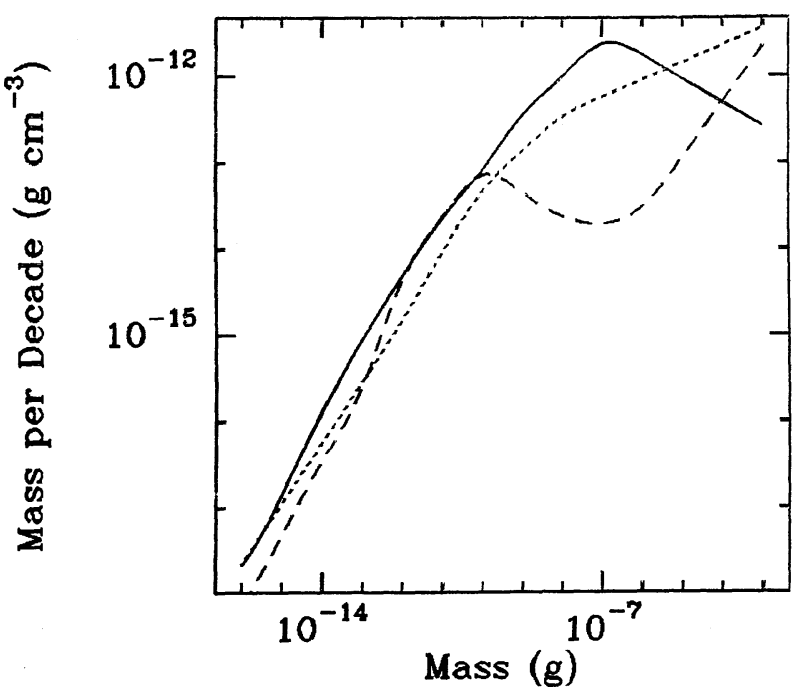

FIG. $3 b$

FiG. 3. - (a) Number of particles per mass decade in the coma of comet Halley as measured in situ by the various space probes. Continuous line: Vega 1 data; short-dashed line: Vega 2 data; long dashed line: Giotto data. (b) Amount of mass per mass decade in the coma of comet Halley as measured in situ by the various space probes. Continuous line: Vega 1 data; short dashed line: Vega 2 data; long dashed line: Giotto data.

are representative of the particle density within a jet, and thus give an overestimate of the average density. From the same figure we deduce that the average particle density is lower by a factor of 2 than the actual density measured by the Vega 1 spacecraft. For the Vega 2 measurements, a factor of 1.5 applies.

\section{MASS ABSORPTION COEFFICIENTS}

\section{a) Optical Constants}

The mass absorption coefficient $\kappa(\lambda)$ and also $\kappa^{\text {eff }}(\lambda)$ depend on the dust material (through the optical constants), the morphology, and the size parameter of the particles. The size parameter is defined as

$$
x=\frac{2 \pi a}{\lambda},
$$

where $a$ is a typical particle dimension. In the case of a sphere, for example, $a$ would be the radius. For the silicates we use the optical constants as presented by Draine (1985) for "astronomical silicates" (see Fig. $4 b$ of this paper). An important feature of this material is its strong $9.7 \mu \mathrm{m}$ band, which we use to model the emission of comet Halley at this wavelength. For the silicate $9.7 \mu \mathrm{m}$ peak absorption, it follows from the above optical constants and a silicate density of $\rho=3.5 \mathrm{~g}$ $\mathrm{cm}^{-3}$ that $\kappa(9.7)=2757 \mathrm{~cm}^{2} \mathrm{~g}^{-1}$ for silicate particles with $x \ll 1$. This value of $\kappa$ is consistent with arguments based on the cosmic abundance of silicates with a mean molecular weight of 150 and an interstellar ratio of silicate absorption to visual extinction of $\left(\tau / A_{V}\right)^{-1}=18.5$ (Roche and Aitken 1984). The value of $2757 \mathrm{~cm}^{2} \mathrm{~g}^{-1}$ is also supported by other observational criteria (Draine and Lee 1984; Chlewicki 1986). The value of $\kappa(9.7)=2000 \mathrm{~cm}^{2} \mathrm{~g}^{-1}$ for amorphous laboratory silicates measured by Day (1979) appears to be somewhat low but is perhaps not out of the question. The upper and lower limits on the $9.7 \mu \mathrm{m}$ absorption strength are also discussed by Hong and Greenberg (1978).

The optical constants of the organic refractories are adopted from the values given by Chlewicki and Greenberg (1990). These are based in part on laboratory measurements of the residues of ice mixtures irradiated with ultraviolet light. The visible properties of the organic refractory are consistent with the observed albedo and linear and circular polarization of silicate core organic refractory interstellar particles. The values of the real and imaginary parts of the refractive index we use are shown in Figure $4 a$, where we have added the absorptivity in the infrared. There are two main features of this material with respect to the present paper: (1) the emission band at 3.4 $\mu \mathrm{m}$, with which we represent the emission feature of comet Halley at this wavelength, and (2) the absorptivity in the visual, which is much higher than the absorptivity of the astronomical silicate in this wavelength region. This higher absorptivity

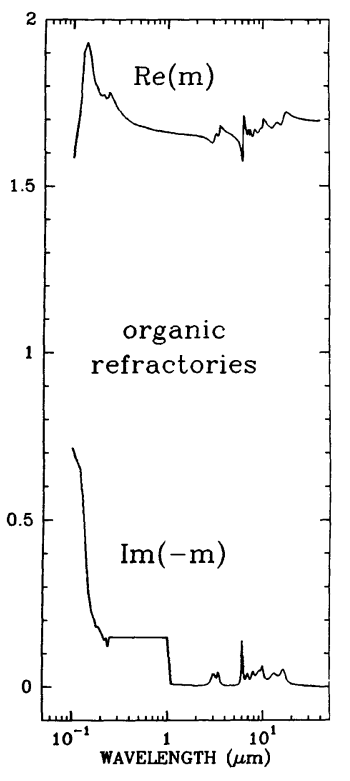

Fig. $4 a$

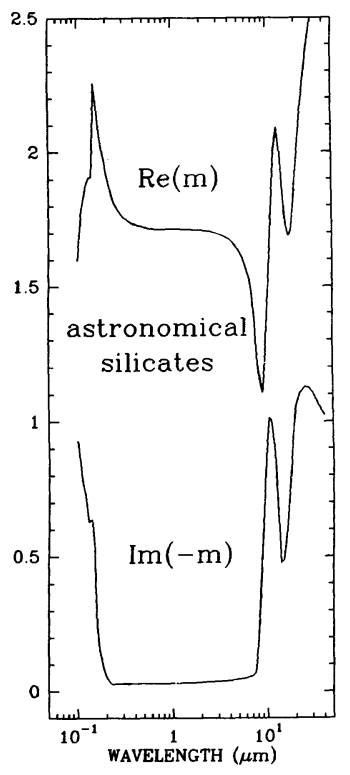

Fig. $4 b$
FIG. 4.-(a) Real (upper curve) and imaginary (lower curve) parts of the refractive index of organic refractory material, as used in this paper. (b) Real (upper curve) and imaginary (lower curve) parts of the refractive index of "astronomical silicates" as given by Draine (1985). 
causes coma dust containing organic refractory material to be hotter than purely silicate dust.

The residues mentioned above represent only the first experimental stage in the photochemical evolution of true Interstellar organic refractories. For the $3.4 \mu \mathrm{m}$ absorption peak the measurement of $\kappa^{\text {eff }}$ for residues is about $800 \mathrm{~cm}^{2} \mathrm{~g}^{-1}$ (Schutte 1988). It is expected that further ultraviolet photoprocessing will lead to a decrease in the number of $\mathrm{CH}_{2}$ and $\mathrm{CH}_{3}$ groups (Greenberg 1982; Schutte 1988). Since these groups are responsible for the $3.4 \mu \mathrm{m}$ band, a reduction in the $3.4 \mu \mathrm{m}$ absorption strength per unit mass results. A value of $\kappa^{\text {eff }}(3.4) \approx 400 \mathrm{~cm}^{2} \mathrm{~g}^{-1}$ appears to be consistent with grain modeling toward the Galactic center (Greenberg 1986c). Some of the most photoprocessed and therefore the most refractory organic mantle material on interstellar dust could have even lower values of $\kappa^{\text {eff }}$. The values we use are, using a density of $\rho=1.8 \mathrm{~g} \mathrm{~cm}^{-3}$ for the organic refractory material, $\kappa(3.4)=554 \mathrm{~cm}^{2} \mathrm{~g}^{-1}$ and $\kappa^{\text {eff }}(3.4)=469 \mathrm{~cm}^{2} \mathrm{~g}^{-1}$.

\section{b) Effect of Size and Shape}

Very large absorbing particles, which satisfy $|m-1| \ll 1$, behave like blackbodies, as shown by van de Hulst (1957). The absorption cross section for such particles is equal to their geometrical surface area, independent of the wavelength. Therefore their efficiency for absorption, $Q_{\text {abs }}$ (defined as $Q_{\text {abs }}=C_{\text {abs }} / G$, where $G$ is the mean geometrical shadow area of the particle), is identically equal to unity, and they show no emission or absorption features. A similar effect may occur for those coma dust particles which are large but do not satisfy $|m-1| \ll 1$, and this is important in conjunction with the 9.7 and $3.4 \mu \mathrm{m}$ emission bands. To illustrate this point, we show in Figure 5 the absorption efficiency of solid silicate spheres for several radii, in the region around $9.7 \mu \mathrm{m}$. Figure 5 shows that in order for a silicate sphere to emit (or absorb) effectively above the continuum at a wavelength $\lambda_{p} \approx 9.7 \mu \mathrm{m}$, at which there is a specific material absorptivity, it must satisfy approximately $a \leq 1 \mu \mathrm{m}$. For the larger spheres the absorption shape is distorted and $\kappa^{\text {eff }}$ is low. This illustrates the important point that, for $\lambda_{p}=9.7 \mu \mathrm{m}$, only those solid silicate particles with a

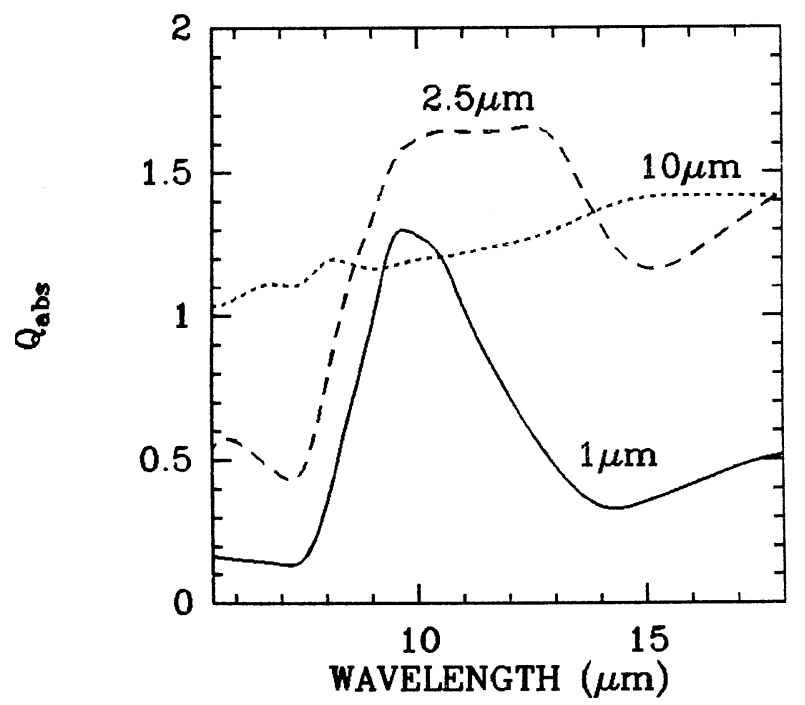

FIG. 5.-Values of the efficiency of absorption for purely silicate spheres. The curves apply to spheres with radii of $1,2.5$, and $10 \mu \mathrm{m}$. Clearly the smaller particles emit the $9.7 \mu \mathrm{m}$ band better than the larger particles. characteristic size smaller than about $1 \mu \mathrm{m}$ will contribute effectively to the $9.7 \mu \mathrm{m}$ excess of the comet emission.

We represent porous aggregates, i.e., coma dust, as a collection of identical particles with a fixed size, which may be either randomly situated and loosely bound to each other or more closely packed. The overall shape of the aggregate is taken as spherical. The parameters describing such an aggregate are the radius $R$ and porosity $P$ of the aggregate, and the refractive index $m$, the typical size $a$, and the shape of the constituent particles. The porosity $P$ is defined by equation (1), where $V$ is defined by the smallest spherical surface which completely surrounds the whole aggregate. As a first approximation we may explore the optical properties of these aggregates by considering them to have the optical characteristics of a cloud of independent particles. This approximation works well for all porosities if $X>10$ ( $X$ being the size parameter of the aggregate). After traversing an optical depth $\tau$, the flux of a plane wave is reduced by a factor $e^{-\tau}$. The average optical depth of a spherical cloud of identical particles is $\tau=$ $(4 / 3) N R C_{\text {ext }}^{\mathrm{i}}$, where $N$ is the number of particles per unit volume, $R$ the radius of the cloud, and $C_{\mathrm{ext}}^{i}$ the cross section for extinction of an individual particle. If the individual particles are small $(x \ll 1)$ and absorbing,

$$
\tau \approx \tau_{\mathrm{abs}}=\frac{4}{3} N R C_{\mathrm{abs}}^{i},
$$

and the absorption cross section of the aggregate is

$$
C_{\mathrm{abs}}=G\left(1-e^{-\tau_{\mathrm{abs}}}\right),
$$

where $G$ is the geometrical cross section of the cloud $\left(G=\pi R^{2}\right)$. We may consider the absorption to be like that of a blackbody if $\tau \gg 1$. In fact, unless $\tau \ll 1$, an increase in $\tau$ will produce a less than proportional change in the absorption cross section because $\tau$ occurs in the exponent. It follows that we can estimate the maximum radius of an aggregate before it behaves like a blackbody as

$$
R_{\max }=\frac{0.5}{N C_{\mathrm{abs}}}=\frac{0.5}{(1-P) \rho \kappa},
$$

so that its maximum mass is given by

$$
M=\frac{4 \pi}{12}[(1-P) \rho]^{-2} \kappa^{-3},
$$

where $\kappa$ and $\rho$ are the mass absorption coefficient and density of the inclusions, respectively.

Equation (18) shows that porous aggregates emit better than equally massive solid ones. For example, an aggregate of density $0.1 \mathrm{~g} \mathrm{~cm}^{-3}$ (a density like that of low-density meteors [Olsson-Steel 1989]), has a porosity $P=0.97$ if its constituent particles have a density like that of silicates, i.e., $\rho_{\text {sil }} \approx 3.5 \mathrm{~g}$ $\mathrm{cm}^{-3}$. According to equation (18), such an aggregate may have a mass of up to $3.3 \times 10^{-8} \mathrm{~g}$ and still emit the $9.7 \mu \mathrm{m}$ feature well. This mass limit is a thousand times higher than the limit of $1.8 \times 10^{-11} \mathrm{~g}$, derived above for solid silicate particles with a radius of $1 \mu \mathrm{m}$.

It is shown by Hage and Greenberg (1990a) that if the inclusions are homogeneous and satisfy $x \ll 1$, the absorption cross sections of the aggregates described above can be determined by calculating an effective refractive index for the whole aggregate and by subsequently using Mie scattering theory to obtain the various cross sections. The effective refractive index of the aggregate, $m_{\mathrm{av}}$, is calculated according to the Maxwell-Garnett 
effective medium theory (Maxwell-Garnett 1904, hereafter Maxwell-Garnett EMT), with vacuum as the so-called matrix material and the individual particles as the "inclusions." The effective refractive index thus obtained depends on the porosity and is given by (Hage and Greenberg 1990a),

$$
m_{\mathrm{av}}^{2}=1+\frac{3(1-P)\left(m^{2}-1\right) /\left(m^{2}+2\right)}{1-(1-P)\left(m^{2}-1\right) /\left(m^{2}+2\right)},
$$

where $m$ is the refractive index of the inclusions. Although it may appear obvious, we note here that it is only correct, for high porosity, to have the vacuum as the matrix, not the solid matter, because the vacuum would, in effect, act like large particles which do not satisfy the Rayleigh condition. Since we assume that the inclusions are individual interstellar dust grains, which at 3.4 and $9.7 \mu \mathrm{m}$ satisfy $x \ll 1$, we may apply the above procedure.

To apply this method also to aggregates consisting of inhomogeneous particles such as interstellar dust grains, we describe these constituent particles as core-mantle spheres with an effective refractive index $m_{\mathrm{av}}^{i}$ determined by the MaxwellGarnett EMT, using the core as "inclusion" and the mantle as "matrix" material. The effective refractive index for the constituent particles we thus obtain is

$$
\left(m_{\mathrm{av}}^{i}\right)^{2}=m_{1}^{2}\left\{1+3 q^{3}\left(\frac{m_{2}^{2}-m_{1}^{2}}{m_{2}^{2}+2 m_{1}^{2}}\right)\left[1-q^{3}\left(\frac{m_{2}^{2}-m_{1}^{2}}{m_{2}^{2}+2 m_{1}^{2}}\right)\right]^{-1}\right\},
$$

where $a$ is the radius of the constituent particle, $q$ and $m_{2}$ are the fractional radius and refractive index of its core, and $m_{1}$ is the refractive index of its mantle. The effective refractive index $m_{\mathrm{av}}^{i}$, given by equation (20), is to be used on the right-hand side of equation (19) to obtain the effective refractive index of the aggregate as a whole. This approach is justified because the polarizability of the core-mantle spheres (which completely determines their scattering properties) implied by equation (20) is exactly correct for concentric core-mantle spheres in the limit $x \rightarrow 0$. This can be seen by comparing equation (20) with the expression, given by van de Hulst (1957) for the polarizability of concentric spheres with $x \ll 1$.

As an example, Figure $6 a$ shows $\kappa^{\text {eff }}(9.7)$ of the coma dust particles as a function of their porosity for various sizes. We have used $\lambda_{1}=7.8 \mu \mathrm{m}$ and $\lambda_{2}=12.5 \mu \mathrm{m}$ (cf. eq. [12]). The mass ratio of silicates to organic refractory material was taken as $2: 1$. A single curve corresponds to equal-mass particles, and the labels correspond to the aggregate radii at $P=0$. For example, an aggregate with porosity $P$ containing the same amount of material as a solid aggregate with a radius $R$ has a radius $a=R /(1-P)^{1 / 3}=32 \mu \mathrm{m}$ for $P=0.97$ and $R=10 \mu \mathrm{m}$. At a given size (but greater than approximately $2 \mu \mathrm{m}), \kappa^{\text {eff }}(9.7)$ increases as the porosity increases. In the limit $P \rightarrow 1$, all aggregates have an effective mass absorption coefficient as high as that of a small particle. Furthermore, for sufficiently high porosities, the shape of the absorption as a function of wavelength is like that of a small particle and not distorted as in Figure 5 (see Hage and Greenberg 1990a). Figure $6 b$ shows $\kappa^{\text {eff }}(9.7)$ of the coma dust particles as a function of their size for $P=0$ and $P=0.99$. Clearly, at a fixed porosity, $\kappa^{\text {eff }}(9.7)$ decreases as the particle size increases. Note how rapidly the effective absorption drops for solid particles $(P=0)$ : from $10^{-14}$ to $2 \times 10^{-10} \mathrm{~g}$ the reduction is about $\frac{1}{2}$, and for $10^{-8} \mathrm{~g}$, $\kappa^{\text {eff }} \approx 0$.

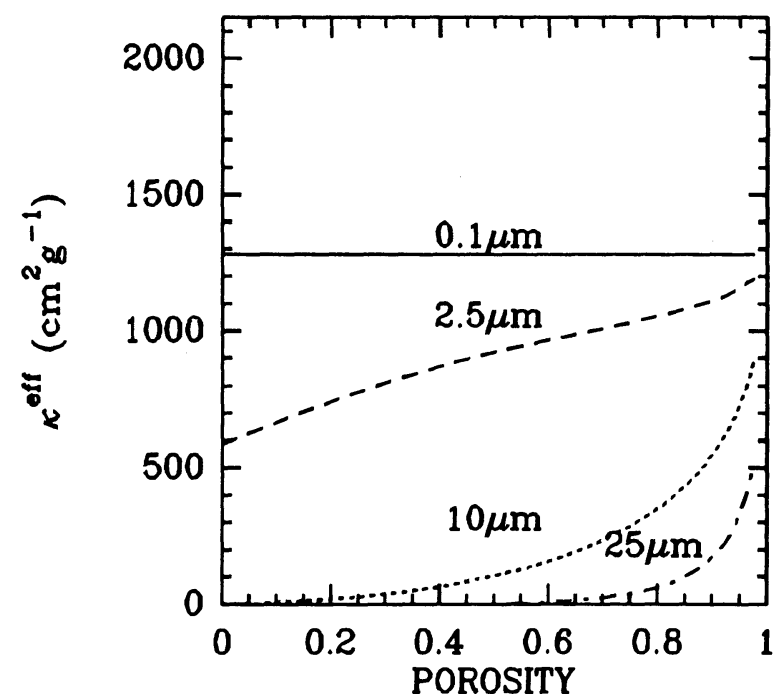

FIG. $6 a$

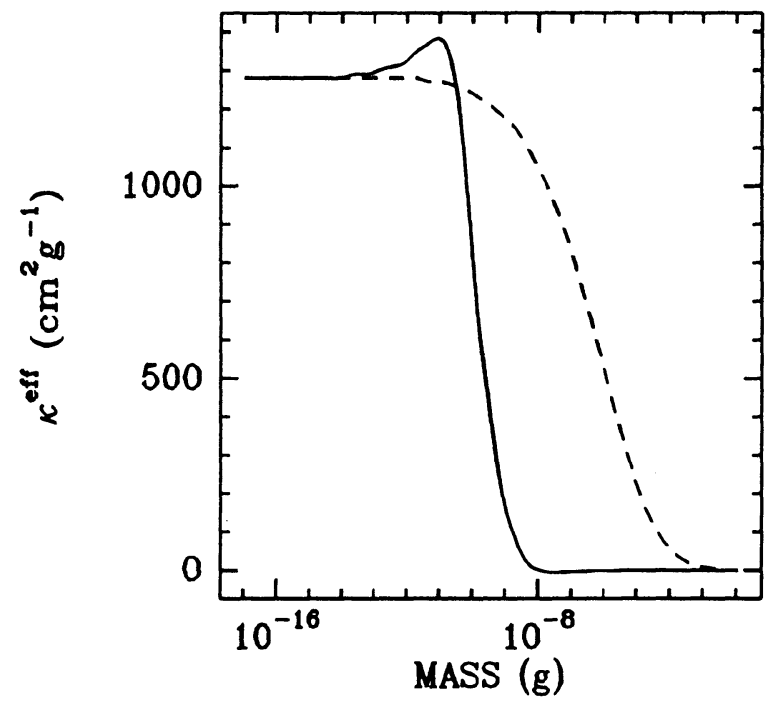

FIG. $6 b$

FIG. 6. - (a) Mass absorption coefficient, $\kappa^{\text {eff }}$, at $\lambda=9.7 \mu \mathrm{m}$ for coma dust as a function of dust porosity and size, assuming $M_{\mathrm{sil}}: M_{\mathrm{or}}=2: 1$. Labels indicate the radius of the aggregate at zero porosity. Each curve applies to a particle of constant mass. (b) Mass absorption coefficient, $\kappa^{\text {eff }}$, at $\lambda=9.7 \mu \mathrm{m}$ for coma dust as a function of the dust size, for two different porosities, assuming $M_{\mathrm{sil}}: M_{\mathrm{or}}=2: 1$. Continuous line: $P=0$; dashed line: $P=0.99$.

\section{DUST TEMPERATURES}

We have used the following standard equation to calculate the temperature of a spherical dust particle in a radiation field

$$
\int_{0}^{\infty} C_{\text {abs }}(\lambda) F_{r}(\lambda) d \lambda=4 \pi \int_{0}^{\infty} C_{\text {abs }}(\lambda) B(\lambda, T) d \lambda
$$

Here $F_{r}$ denotes the ambient radiation field, and $C_{\mathrm{abs}}$ and $T$ are the absorption cross section and temperature of the dust particle, respectively. Equation (21) expresses the fact that, in equilibrium with the radiation field, a body emits the same amount of energy as thermal emission as it absorbs at other wavelengths. Let us first mention a few special limiting cases: (1) absorbing particles which are relatively large will have $Q_{\text {abs }}(\lambda) \approx 1$ for a large range in $\lambda$, so that they will have approx- 
imately the temperature of a blackbody, $T \approx 279 r^{-0.5}$, where $r$ is the distance to the Sun, in astronomical units; (2) individual core-mantle dust particles with a typical size of, e.g., a few tenths of a micron will be much hotter, because they emit relatively poorly in the infrared (since $C_{\mathrm{abs}} \sim a^{3} / \lambda$ ) but absorb well in the visual and ultraviolet; (3) last, the temperature attained by an aggregate in the limit $P \rightarrow 1$ is equal to the temperature of a single inclusion as if it were exposed to the radiation field on its own. This is because the inclusions in very porous aggregates do not shadow each other much and absorb and emit radiation nearly as if they are independent of one another. In general, the temperature of an aggregate depends on its distance to the Sun, on its size and porosity, and on the properties of the individual constituents.

To satisfy equation (21) for a certain particle in practice, one has to calculate the emitted power for a range of temperatures, calculate the absorbed power, and then interpolate to match emission and absorption and find the correct particle temperature (see, e.g., Greenberg 1971). To calculate the absorption cross sections for the solid particles, we have used the standard Mie theory. For solid core-mantle particles we have used another appropriate version of the Mie theory. As far as the aggregates are concerned, we may only use the combined Mie theory-Maxwell-Garnett EMT approach to calculate their absorption cross sections if $x \ll 1$ for the individual constituents. Since the individual constituents are interstellar dust grains with a radius of about $0.1 \mu \mathrm{m}$, the above approach is valid up to $\lambda \approx 1 \mu \mathrm{m}$. For shorter wavelengths we have used equation (16). This approximation works well if $X>10$.

As an example, results for the temperatures of various aggregates as a function of porosity and size are shown in Figure 7. The mass ratio of silicates to organic refractory material was taken as $2: 1$. The temperatures shown are computed for a solar distance of $0.9 \mathrm{AU}$. In general, the trends discussed above are borne out by the results of the exact calculations used to draw Figure 7. Note that for large particles the temperature does not rise rapidly to the limiting value until quite high values of $P$.

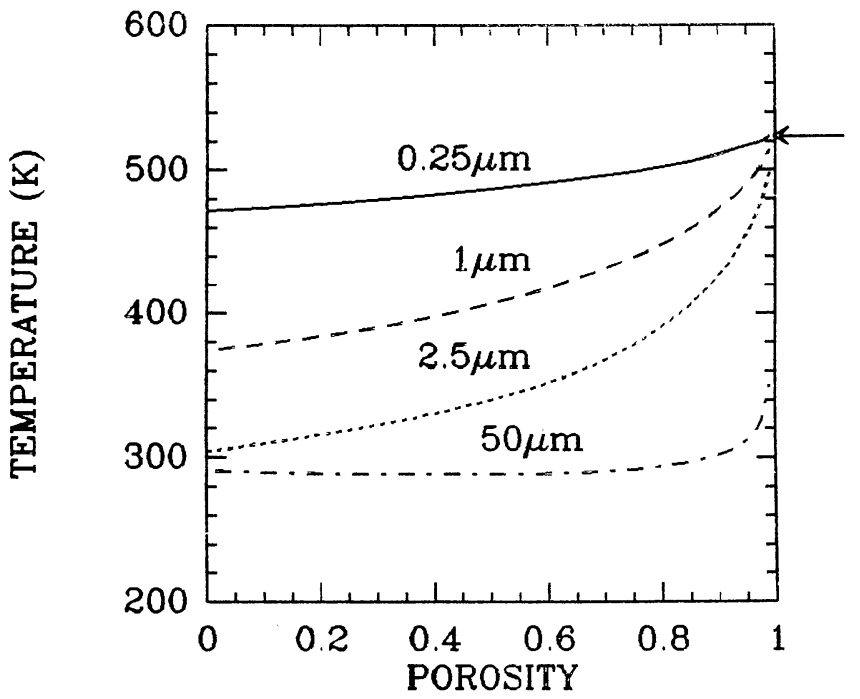

Fig. 7.- Temperature of coma dust as a function of dust porosity and size, assuming $M_{\mathrm{si} 1}: M_{\mathrm{or}}=2: 1$, at a distance of $0.9 \mathrm{AU}$ from the Sun. Labels indicate the radius of the aggregate at zero porosity. Each curve applies to a particle of constant mass. The arrow indicates the limiting temperature corresponding to that of a single interstellar grain.

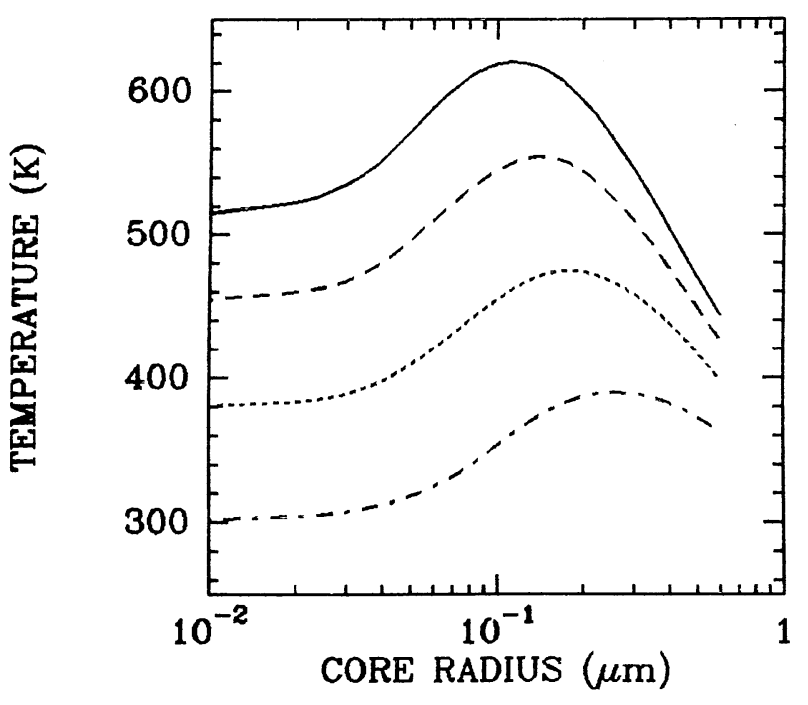

Fig. 8.-Temperatures of individual, spherical core-mantle interstellar grains as a function of their size, at a distance of $0.9 \mathrm{AU}$ from the Sun. The various curves correspond to different thicknesses of the organic refractory mantles. Top curve: $M_{\mathrm{sil}}: M_{\text {or }}=1: 1$; long-dashed curve: $M_{\mathrm{sil}}: M_{\mathrm{or}}=2: 1$; short-dashed curve: $M_{\mathrm{sil}}: M_{\mathrm{or}}=5: 1 ;$ dash-dot curve: $M_{\mathrm{sil}}: M_{\mathrm{or}}=1: 0$.

This limiting temperature is determined by the size and morphology of the individual inclusions. As shown in Figure 8, the highest limiting temperatures are given by particle inclusions of interstellar dust size.

\section{RESULTS}

In this section we show results for the theoretical amount of excess thermal emission above the continuum, at 9.7 and 3.4 $\mu \mathrm{m}$, from the coma of comet Halley as a function of the porosity of the dust, obtained by combining the methods presented in $\$$ III-V. The integration over mass (cf. eq. [13]) was done from zero mass up to a mass of about $0.1 \mathrm{~g}$. At this mass, the integral on the left-hand side of equation (13) has converged, in spite of the fact that more mass is present in the higher ranges than in the lower ranges. This is because of the decrease of $\kappa^{\text {eff }}$ and $T$ as the particle mass increases (cf. Figs. $6 b$ and 7). By equating these theoretical values with results of ground-based observations, we have derived the porosity of the coma dust. Subsequently, we have used the values of the porosity of the coma dust derived in this way to calculate the theoretically expected shape of the $9.7 \mu \mathrm{m}$ emission band $(7.8 \mu \mathrm{m}<\lambda<12.5$ $\mu \mathrm{m})$, to be compared with the observed shape.

As mentioned before, theoretical values of the emission by the coma which are calculated based on the dust size spectrum obtained on a certain spacecraft must be compared only with those ground-based observations which have been done close in time to the measurements by this spacecraft. Figure 9 shows the theoretical excess emission at $9.7 \mu \mathrm{m}$ from comet Halley's coma in an aperture corresponding to $5620 \mathrm{~km}$ at the comet nucleus. The theoretical emission was calculated based on comet Halley's position on March 6.85 (comet-Sun distance $r=0.8 \mathrm{AU}, \Delta=1.14 \mathrm{AU}$ ) and using the particle-mass spectrum measured by Vega 1 on March 6 . These theoretical values are to be compared with the ground-based observations on March 6.85 (dashed horizontal line). The four different curves shown in Figure 9 correspond to four different assumed values of the silicate-to-organics mass ratio in the coma dust. From the top downward, the curves correspond to mass ratios of 


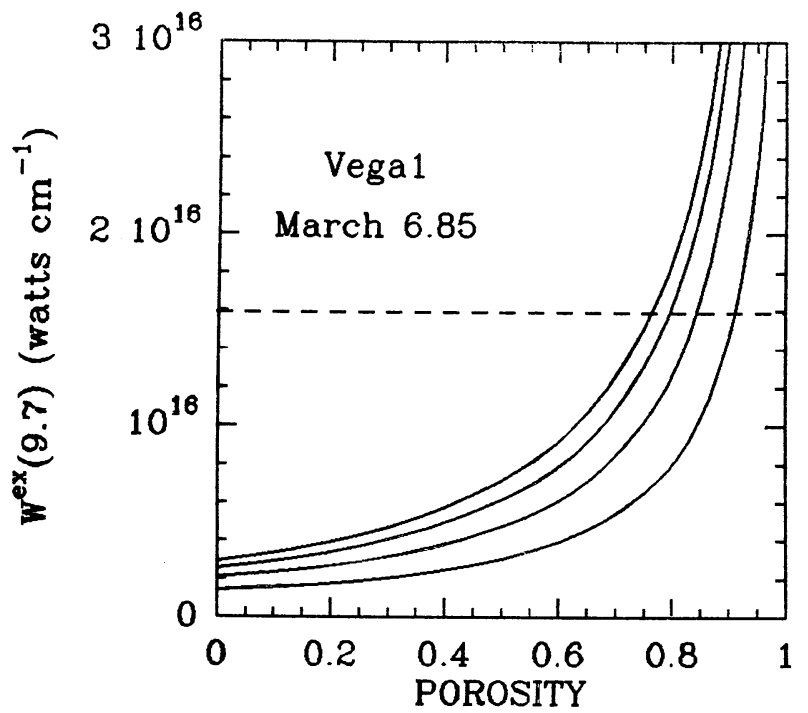

Fig. 9.-Power per unit wavelength at $9.7 \mu \mathrm{m}$ emitted from coma of comet Halley calculated using eq. (13), as a function of the porosity of the coma dust. The calculations correspond to the power emitted from the spherical region around the nucleus with a radius of $5620 \mathrm{~km}$ on March 6.85 , and use the particle-size distribution measured by $V e g a l$. The various curves correspond to different mass ratios of silicates to organic refractory material in the coma dust. From the top curve downward: $M_{\mathrm{sil}}: M_{\mathrm{or}}=1: 1, \quad M_{\mathrm{sil}}: M_{\mathrm{or}}=2: 1$, $M_{\text {sil }}: M_{\text {or }}=5: 1$, and (lowest curve) $M_{\mathrm{sil}}: M_{\mathrm{or}}=1: 0$. The horizontal line denotes the amount of emission as observed from Earth.

silicates to organics of $1: 1,2: 1,5: 1$, and $1: 0$. Figures 10 and 11 show the theoretical emission at $9.7 \mu \mathrm{m}$ from the coma in an aperture corresponding to $4900 \mathrm{~km}$ at the comet nucleus. The theoretical emission was calculated based on comet Halley's position on March $12.8(r=0.89 \mathrm{AU}, \Delta=0.99 \mathrm{AU})$ and using the particle-mass spectrum measured by Vega 2 (Fig. 10) and

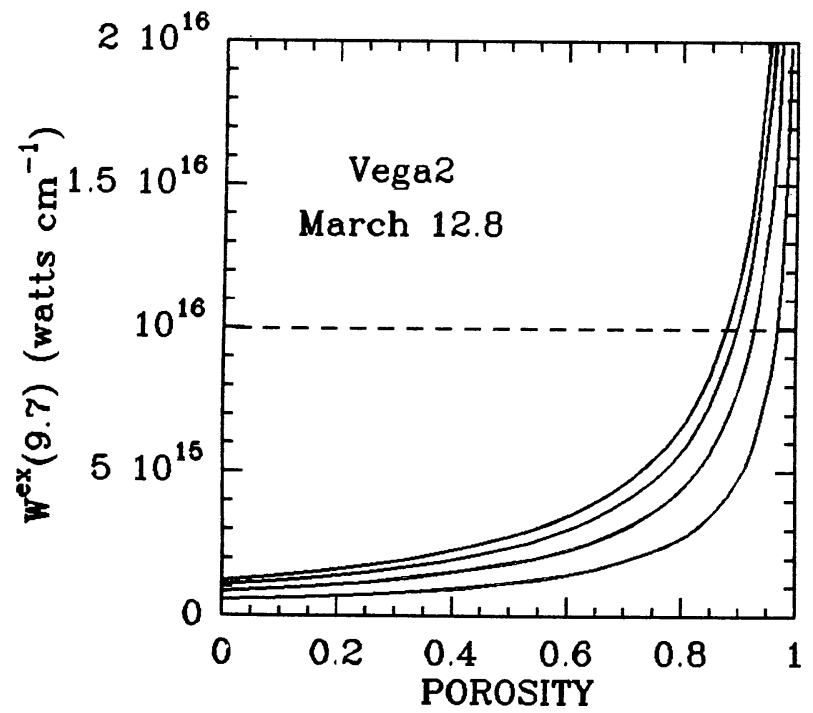

FIG. 10.-Power per unit wavelength at $9.7 \mu \mathrm{m}$ emitted from coma of comet Halley calculated using eq. (13), as a function of the porosity of the coma dust. The calculations correspond to the power emitted from the spherical region around the nucleus with a radius of $4900 \mathrm{~km}$ on March 12.8, and use the particle-size distribution measured by Vega 2 . The various curves correspond to different mass ratios of silicates to organic refractory material in the coma dust. From the top curve downward: $M_{\mathrm{sil}}: M_{\mathrm{or}}=1: 1, M_{\mathrm{sil}}: M_{\mathrm{or}}=2: 1$, $M_{\mathrm{sil}}: M_{\mathrm{or}}=5: 1$, and (lowest curve) $M_{\mathrm{sil}}: M_{\mathrm{or}}=1: 0$. The horizontal line denotes the amount of emission as observed from Earth.

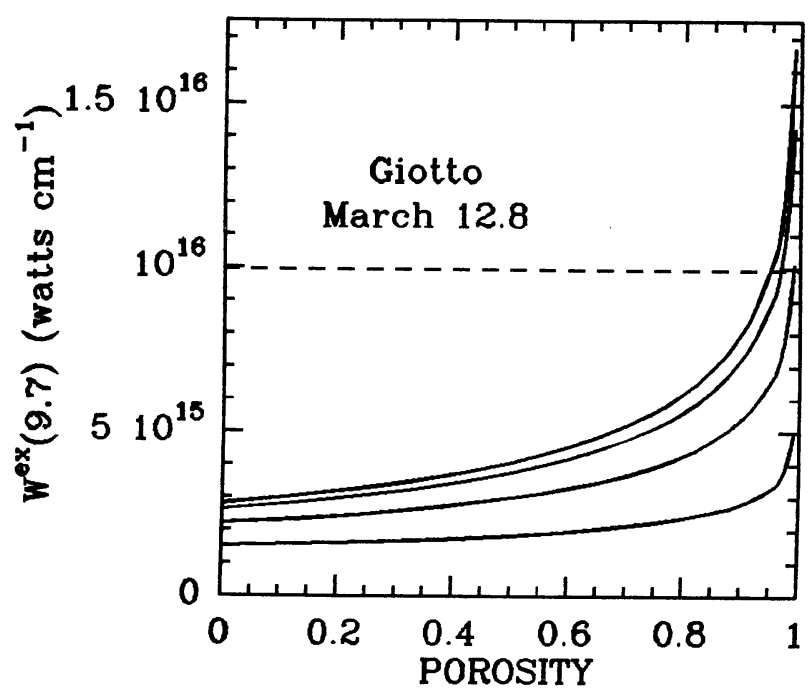

Fig. 11.-Power per unit wavelength at $9.7 \mu \mathrm{m}$ emitted from coma of comet Halley calculated using eq. (13), as a function of the porosity of the coma dust. The calculations correspond to the power emitted from the spherical region around the nucleus with a radius of $4900 \mathrm{~km}$ on March 12.8, and use the particle-size distribution measured by Giotto. The various curves correspond to different mass ratios of silicates to organic refractory material in the coma dust. From the top curve downward: $M_{\mathrm{si1}}: M_{\mathrm{or}}=1: 1, \quad M_{\mathrm{sil}}: M_{\mathrm{or}}=2: 1$, $M_{\mathrm{sil}}: M_{\mathrm{or}}=5: 1$, and (lowest curve) $\boldsymbol{M}_{\mathrm{si1}}: M_{\mathrm{or}}=1: 0$. The horizontal line denotes the amount of emission as observed from Earth.

Giotto (Fig. 11). These theoretical values are to be compared with the ground-based observations on March 12.8 (dashed horizontal line). From the top downward, the curves correspond to mass ratios of $1: 1,2: 1,5: 1$, and $1: 0$, in both figures. Figures 12,13, and 14 show the same set of curves as in Figures 9,10 , and 11 , respectively, but for the emission at $3.4 \mu \mathrm{m}$.

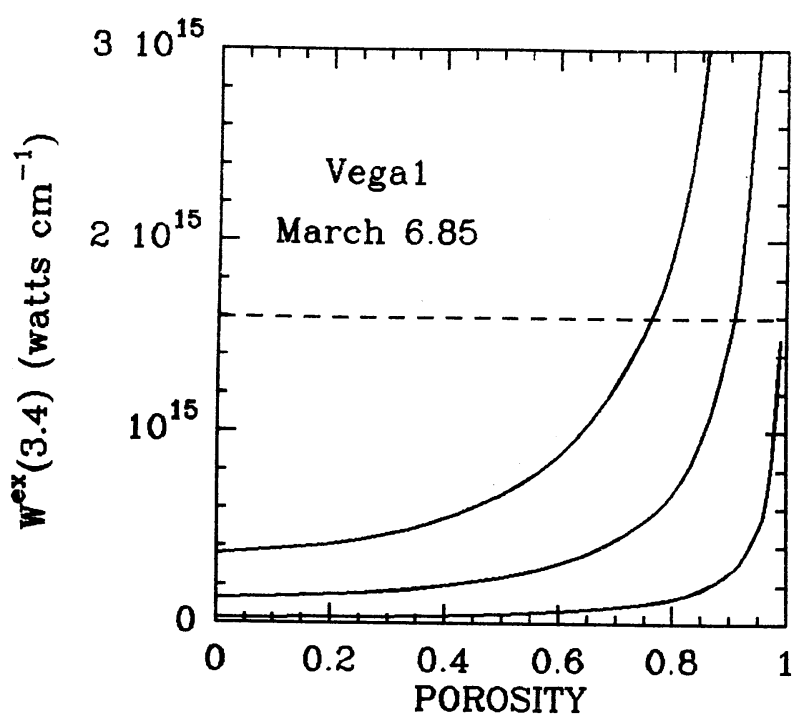

Fig. 12.-Power per unit wavelength at $3.4 \mu \mathrm{m}$ emitted from coma of comet Halley calculated using eq. (13), as a function of the porosity of the coma dust. The calculations correspond to the power emitted from the spherical region around the nucleus with a radius of $5620 \mathrm{~km}$ on March 6.85 , and use the particle-size distribution measured by Vega 1 . The various curves correspond to different mass ratios of silicates to organic refractory material in the coma dust. From the top curve downward: $M_{\mathrm{sil}}: M_{\mathrm{or}}=1: 1, M_{\mathrm{sil}}: M_{\mathrm{or}}=2: 1$, and (lowest curve) $M_{\mathrm{sil}}: M_{\mathrm{or}}=5: 1$. The horizontal line denotes the amount of emission as observed from Earth. 


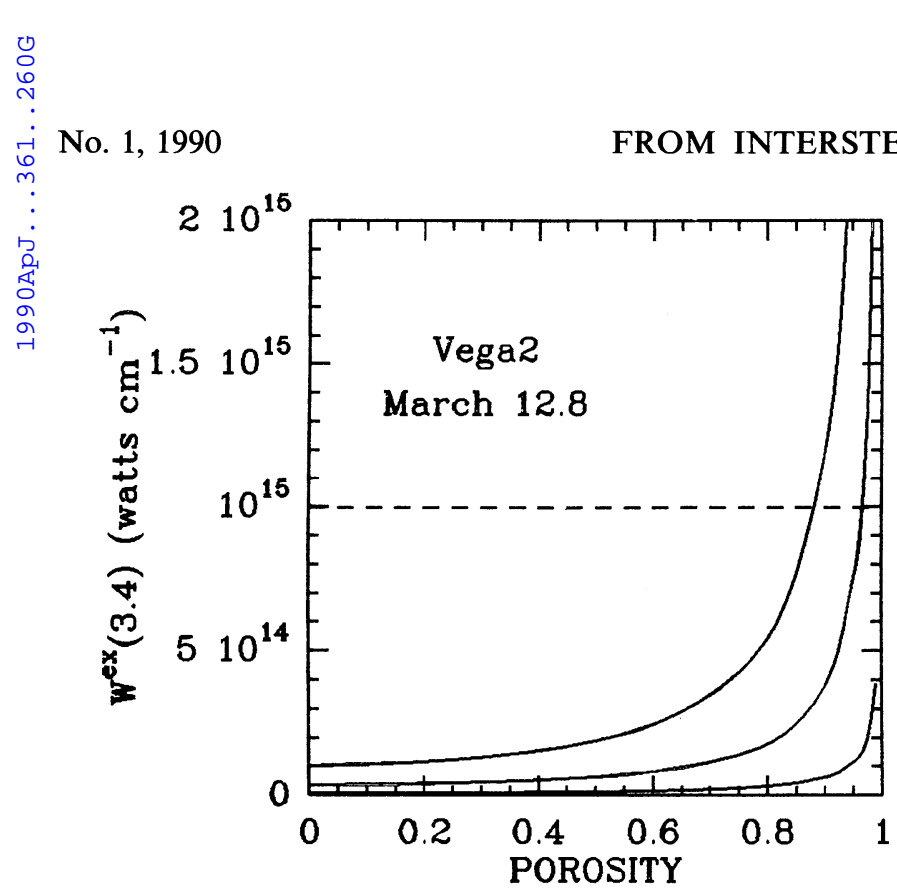

Fig. 13.-Power per unit wavelength at $3.4 \mu \mathrm{m}$ emitted from coma of comet Halley calculated using eq. (13), as a function of the porosity of the coma dust. The calculations correspond to the power emitted from the spherical region around the nucleus with a radius of $4900 \mathrm{~km}$ on March 12.8, and use the particle-size distribution measured by Vega 2 . The various curves correspond to different mass ratios of silicates to organic refractory material in the coma dust. From the top curve downward: $M_{\mathrm{sil}}: M_{\mathrm{or}}=1: 1, M_{\mathrm{sil}}: M_{\mathrm{or}}=2: 1$, and (lowest curve) $M_{\mathrm{sil}}: M_{\mathrm{or}}=5: 1$. The horizontal line denotes the amount of emission as observed from Earth.

We have used the observed values of $F^{\text {ex }}(9.7)$ and the assumed ratio of $F^{\mathrm{ex}}(9.7) / F^{\mathrm{ex}}(3.4)=10$ to read the required porosities from these figures, in order to satisfy equation (13). Table 2 shows the results obtained, including results of similar calculations and observations on March 13.75.

After having thus found the porosity necessary to fit the

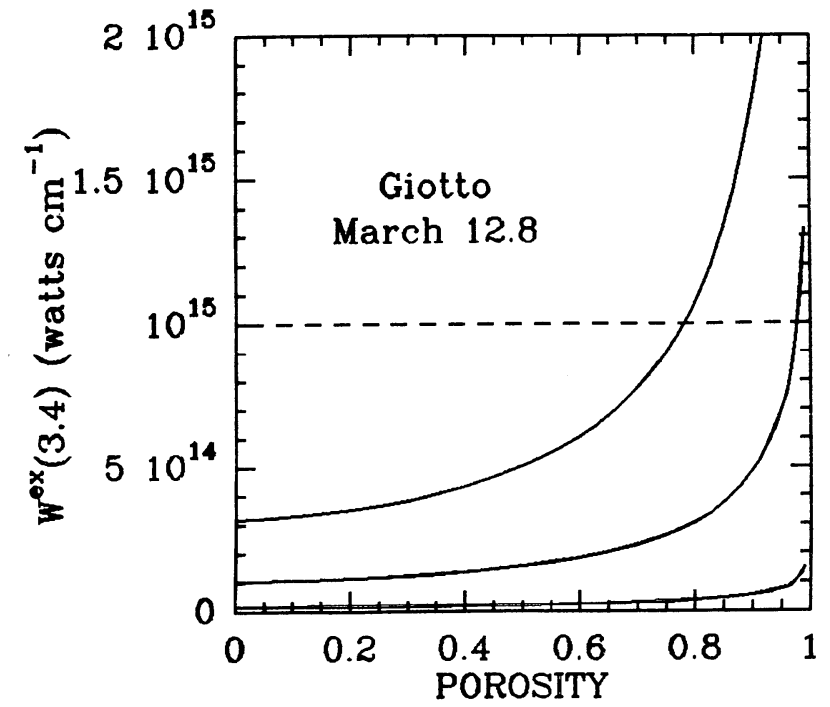

Fig. 14.-Power per unit wavelength at $3.4 \mu \mathrm{m}$ emitted from coma of comet Halley calculated using eq. (13), as a function of the porosity of the coma dust. The calculations correspond to the power emitted from the spherical region around the nucleus with a radius of $4900 \mathrm{~km}$ on March 12.8, and use the particle-size distribution measured by Giotto. The various curves correspond to different mass ratios of silicates to organic refractory material in the coma dust. From the top curve downward: $M_{\mathrm{sil}}: M_{\mathrm{or}}=1: 1, M_{\mathrm{sil}}: M_{\mathrm{or}}=2: 1$, and (lowest curve) $M_{\mathrm{sil}}: M_{\mathrm{or}}=5: 1$. The horizontal line denotes the amount of emission as observed from Earth.
TABLE 2

Porosity of Coma Dust Derived from Equation (13) ${ }^{\mathrm{a}}$

\begin{tabular}{ccccc}
\hline \hline$M_{\text {sil }}: M_{\text {or }}$ & $\begin{array}{c}\text { March 6.85 } \\
\text { Vega 1 }\end{array}$ & $\begin{array}{c}\text { March 12.8 } \\
\text { Vega 2 }\end{array}$ & $\begin{array}{c}\text { March 12.8 } \\
\text { Giotto }\end{array}$ & $\begin{array}{c}\text { March 13.75 } \\
\text { Giotto }\end{array}$ \\
\hline \multicolumn{5}{c}{$P(9.7)$} \\
$1: 1 \ldots \ldots \ldots$ & 0.76 & 0.87 & 0.95 & 0.81 \\
$2: 1 \ldots \ldots \ldots$ & 0.80 & 0.90 & 0.97 & 0.84 \\
$5: 1 \ldots \ldots \ldots$ & 0.84 & 0.93 & 0.99 & 0.94 \\
$1: 0 \ldots \ldots \ldots$ & 0.91 & 0.97 & $>0.99$ & $>0.99$ \\
\hline & & $P(3.4)$ & & \\
\hline $1: 1 \ldots \ldots \ldots$ & 0.76 & 0.87 & 0.78 & 0.64 \\
$2: 1 \ldots \ldots \ldots$ & 0.90 & 0.96 & 0.98 & 0.95 \\
$5: 1 \ldots \ldots \ldots$ & $>0.99$ & $>0.99$ & $>0.99$ & $>0.99$ \\
\hline
\end{tabular}

$P(9.7)$ is the porosity required to match the strength of the $9.7 \mu \mathrm{m}$ emission band, and $P(3.4)$ is the result for the $3.4 \mu \mathrm{m}$ band. The entry $>0.99$ means that the observed band strength cannot be matched with a realistic porosity, for that particular combination of assumed size distribution and mass ratio of silicate to organic refractory.

amount of excess emission, it is possible to compute the expected shape of the $9.7 \mu \mathrm{m}$ band (cf. eq. [9]), using the values for the porosity listed in Table 2 . For example, the curve in Figure 15 shows the result for the band shape on March 12.8, using a porosity of about 0.97 and the Giotto size distribution. The crosses in this figure represent ground-based observations on this date, by Hanner et al. (1987). Figure 15 shows that a reasonable match is obtained. However, using $P \approx 0.80$ and the Vega 1 mass spectrum, the resulting bandwidth is about $50 \%$ too wide compared with the observations by Hanner et al. on March 6.85 (see Fig. 16).

\section{DISCUSSION}

\section{a) Discussion of the Results}

From Table 2 we can immediately conclude that, using a silicate-to-organics mass ratio of $1: 1$ or $2: 1$, the strengths of the 3.4 and $9.7 \mu \mathrm{m}$ bands can be reproduced theoretically, on

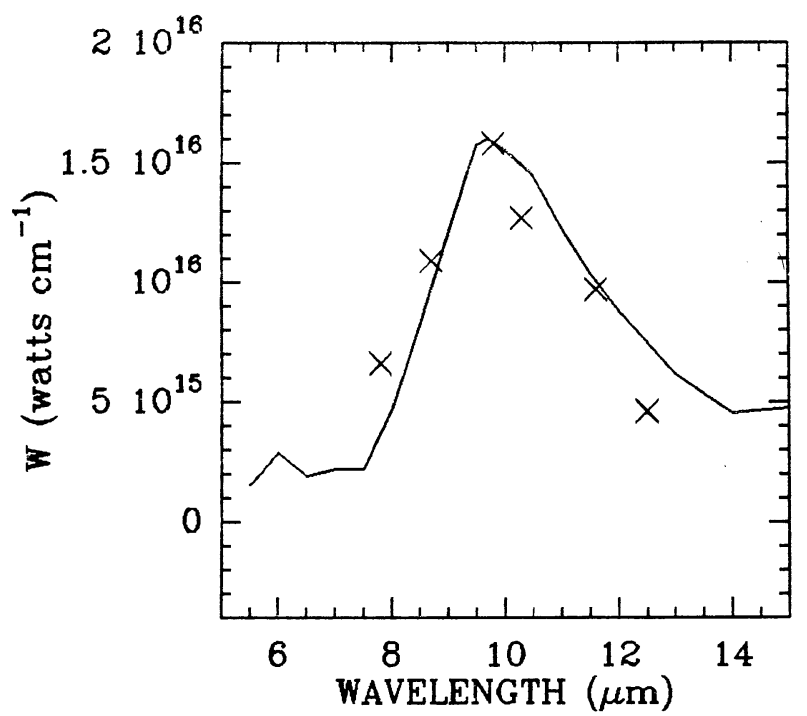

FIG. 15.-Shape of the $9.7 \mu \mathrm{m}$ band calculated using eq. (9), using the Giotto dust size distribution and a porosity of $P \approx 0.97$. The crosses show the observations by Hanner et al. (1987) on March 12.8. The curve is made to go through the observational data point at $9.7 \mu \mathrm{m}$. 


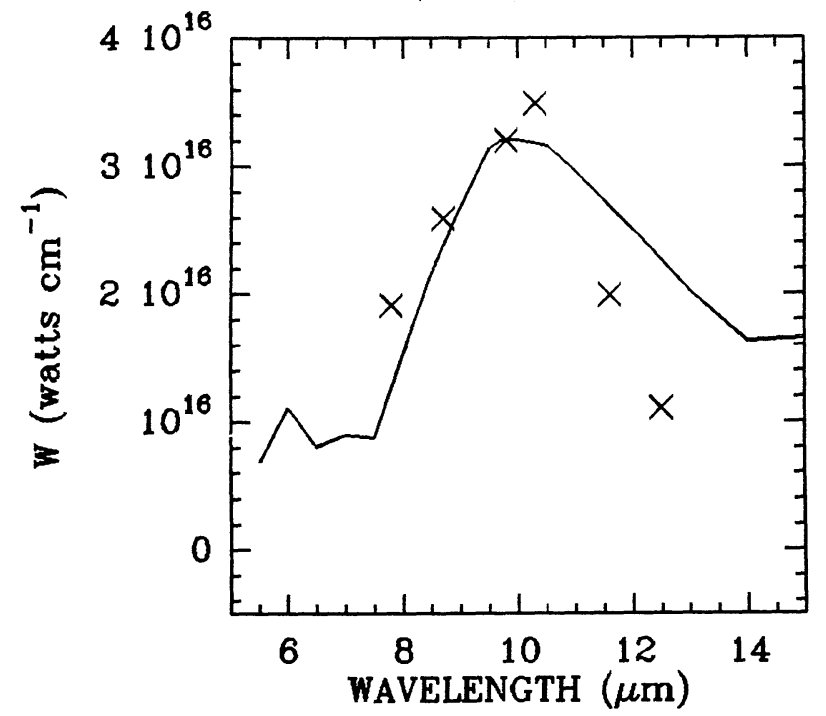

FIG. 16.- Shape of the $9.7 \mu \mathrm{m}$ band calculated using eq. (9), using the Vega 1 dust size distribution and a porosity of $P \approx 0.80$. Crosses show the observations by Hanner $e$ t al. (1987) on March 6.85. The curve is made to go through the observational data point at $9.7 \mu \mathrm{m}$.

the basis of the concept of porous aggregates of interstellar dust. The observed strengths of these bands are inconsistent when using purely silicate dust, or using a silicate to organics mass ratio of $5: 1$. We therefore discount these mass ratios as likely possibilities, and leave them out of the rest of the discussion. Coma dust porosities which are in all cases significantly higher than zero are required. The porosities required to match both the 3.4 and the $9.7 \mu \mathrm{m}$ bands simultaneously, using either the 1:1 mass ratio or the $2: 1$ mass ratio, are slightly discrepant. This is not surprising in view of the uncertainty in the observational value of the $3.4 \mu \mathrm{m}$ excess emission. Rather, the fact that the required porosities $P(3.4)$ and $P(9.7)$ are in most cases rather similar and simultaneously significantly higher than zero gives further confidence in the consistency of the model. The mass ratio of silicates to organics in the coma dust, measured in situ, was approximately 2:1 (Kissel and Krueger 1987). In order to be able to satisfy this additional observational constraint, we will use only the results for this mass ratio in the following. We recall from Table 1 that for this mass ratio, a lower limit on the coma dust porosity of $P=0.83$ is implied by the interstellar dust model of comets. Table 2 shows that the required porosities using the Vega 1 March 6.85 data and the Giotto March 13.75 data are approximately consistent with this limit, implying that the comet nucleus has a zero porosity. On the other hand, the porosities derived with the Vega 2 and Giotto mass spectra on March 12 seem to point more toward a higher nucleus porosity, more in keeping with what was derived for the nucleus density by Sagdeev, Elyasberg, and Moroz (1988) or Sekanina and Yeomans (1985).

The above discussion does not take into account possible errors or uncertainties in the observations and calculations. For example, one might expect $\kappa^{\text {eff }}(3.4)$ to be a function of temperature because the organic refractory consists of many different compounds which individually have different evaporation rates and band strengths. The more refractory components would have the lower band strengths which would lead to higher porosities, all other things being equal. Because of the complexity of the calculations involving equation (13), it would be difficult and very time-consuming to establish the effect of variations in the various parameters on the final result. Rather, the approach of this work has been to choose the most reasonable parameter values and physical models to derive the end result. We may investigate the effect of a hypothetical systematic error which causes the observational value to go either up or down by a factor of 2 relative to the theoretical values of the coma excess emissions. Considering the data for the $9.7 \mu \mathrm{m}$ band and the $2: 1$ mass ratio, we find by increasing the observational values of $W^{\text {ex }}$ by a factor of 2 the following porosities: $P=0.91$ (Vega 1), $P=0.97$ (Vega 2), $P>0.99$ (Giotto March 12.8), and $P=0.98$ (Giotto March 13.75). These porosities all indicate low comet nucleus densities, consistent with the values derived based on the interstellar dust model of comets. Decreasing $W^{\text {ex }}$ by a factor of 2 , we obtain $P=0.6$ (Vega 1), $P=0.76$ (Vega 2), $P=0.73$ (Giotto March 12.8), and $P=0.27$ (Giotto March 13.75). Within the framework of the interstellar dust model of comets, the first three required porosities are at best only barely consistent with a fully packed comet nucleus. The porosity $P=0.27$ is totally inconsistent with the present comet model and is even much too low for interplanetary dust particles, for which $P \approx 0.7$ (see $\S$ VII $d$ ).

At first sight this exercise seems to leave many possibilities (or should we say impossibilities) open. We must therefore look at the broader picture and consider additional constraints to select the most likely possibility consistent with the present results. An additional observational constraint is the shape of the $9.7 \mu \mathrm{m}$ band. The broader picture involves the consideration of the comet and meteor densities derived by others and a deeper look into the coma dust size spectra observations.

\section{i) The $9.7 \mu \mathrm{m}$ Band Shape}

The shapes of the $9.7 \mu \mathrm{m}$ band, calculated using the porosities listed in the second row of Table 2 and the optical constants for "astronomical silicates," agree fairly well with the observed shapes in the case of the Vega 2 and Giotto size distributions. The shape calculated using the Vega 1 size distribution is significantly too broad. The extra width is due to the low porosity required for the Vega 1 March 6.85 data and the relatively large amount of larger particles in the Vega 1 size distribution. Since a higher porosity would give a narrower band, the observed band shape points in the direction of a higher porosity than derived at present. The observed extra emission at $11.2 \mu \mathrm{m}$, caused by crystalline silicates, is reserved for a later discussion. A preliminary estimate of the $11.2 \mu \mathrm{m}$ band strength using optical constants for crystalline silicates indicates that these constitute only about $5 \%$ of the total silicates in comet Halley (Greenberg, Zhao, and Hage 1989), so that this extra emission may be ignored for the present discussion.

\section{ii) The Coma Dust Size Distributions}

The size distributions we have used may be subject to revision because calibration of the measuring instruments has been based on experience with compact particles rather than with porous particles. This possibility has already been suggested for the Vega data by Smirnov, Vaisberg, and Anasimov (1987), where it is stated, "If cometary particles are made in a complicated manner as suggested by Greenberg (1982) or if they are similar to Brownlee particles (Brownlee 1978) it is possible that penetration processes are different for this kind of projectiles." Moreover, the presence in the Vega size distribution of a larger number of particles with $M>10^{-11} \mathrm{~g}$ relative to the Giotto size distribution results not only in a generally lower range of porosities in Table 2 but also in predicting 
significantly higher dust-to-gas ratios. Using a gas emission rate of $2.55 \times 10^{7} \mathrm{~g} \mathrm{~s}^{-1}$ for Halley at the time of the Giotto encounter (Krankowsky et al. 1986), the dust-to-gas ratio including dust particles up to a limiting mass of $10^{-5} \mathrm{~g}$ in the Giotto size distribution gives $\left(M_{d} / M_{g}\right)_{<10^{-5}}^{G}=0.2$. For the same gas emission rate the Vega 1 data give $\left(M_{d} / M_{g}\right)_{<10^{-5}}^{V 1}=$ 4 , and the Vega 2 data give $\left(M_{d} / M_{g}\right)_{<10^{-5}}^{V 2}=2$, both of which are already higher than the value 1 (an approximate value based on solar abundance) even though the contributions of the higher mass particles have not been included. According to McDonnell et al. (1989), the dust-to-gas ratio up to $\approx 10^{-2} \mathrm{~g}$ gives a value $\left(M_{d} / M_{g}\right)_{<10^{-2}}^{G} \approx 1.3$, so that the total mass between $10^{-5}$ and $10^{-2} \mathrm{~g}$ is about 5 or 6 times larger than from 0 to $10^{-5} \mathrm{~g}$. Applying a similar factor at the time of the $V e g a$ encounters would imply dust-to-gas ratios of at least 20 in the case of Vega 1 and 10 in the case of Vega 2. We might bring these values down if we take into account possible shortterm variations by a factor of about 2 in the water production rate between the Giotto and Vega encounters as recorded by Feldman et al. (1987). But even using a 2 times higher water production rate, the predicted dust-to-gas production ratios for the Vega spectra are unacceptably high, since the solar abundance limitation on the mean dust-to-gas ratio in the comet nucleus is only $\left(M_{d} / M_{g}\right)=0.82$. Of course we have to accept the possibility that over short time spans the dust and gas need not be totally coupled in their ejection from the nucleus. Perhaps there could be events which bring off a fair amount of crustal dust (from which the volatiles have already evaporated). But then why would this have occurred for both the Vega 1 and the Vega 2 cases and not for the Giotto case? We conclude that there are reasons to question the Vega dust size distribution spectra for $M>10^{-11} \mathrm{~g}$ and that even the Giotto size distribution may be a little high in some size ranges. Since the dust-to-gas ratios predicted by the mass spectra are on the high side compared with solar abundance, it seems likely that the mass spectra we have used overestimate the amount of mass in some ranges. This leads again to the conclusion that some of the derived porosities in Table 2 are more likely to be underestimated than overestimated. Finally, we note that using the unrevised Giotto size distribution, given by McDonnell et al. (1987), it was not possible to fit the $9.7 \mu \mathrm{m}$ excess emission on March 12.8 , and a very high porosity was needed to fit the band on March 13.75.

iii) Comet Nucleus Densities and Meteor Densities

Sagdeev et al. (1988), Sekanina and Yeomans (1985), and Rickman et al. (1988) derived comet nucleus densities of 0.6, 0.25 , and $<0.5 \mathrm{~g} \mathrm{~cm}^{-3}$. The derivation in $\S$ II $b$ has shown that these densities imply coma dust porosities of $P \geq 0.933$, within the framework of the present comet model. Additionally, high coma dust porosities are consistent with the observed low meteor densities, as was also shown in $\S \mathrm{II} b$.

We conclude, therefore, that a high coma dust porosity of $0.93 \leq P \leq 0.975$ is a likely possibility which is consistent with (a) the direct results of this work, $(b)$ comet densities as deduced independently by other workers, and (c) observed meteor densities. Furthermore, we are led to conclude that, in addition to the required high dust porosity, there are two basic features in the interstellar dust model of comets which are absolutely critical in producing the observed 9.7 and $3.4 \mu \mathrm{m}$ coma dust emissions:

1. Heating by the organic refractory material.-Without heating of the coma dust by the organic refractory material, it is in some cases impossible to reproduce the amount of excess $9.7 \mu \mathrm{m}$ emission, even with a very high porosity $(1-P \ll 1)$, as shown in Table 2 . While it is true in principle that the organic refractory material could be present as a separate component in contact with the silicates but not necessarily as mantles, the interstellar dust model naturally provides both the needed relative amounts and the necessary thermal contact. Further confirmation of this core-mantle morphology is given by Jessberger (1989), based on the different initial velocities of the CHON ions and the ions of the rock-forming elements after impact in the mass spectrometer. Incidentally, the fact that a large fraction of the carbon in the comet is contained in the organic refractory mantles makes it understandable why the carbon-to-oxygen ratio in the coma gas constituents is about half of the solar abundance ratio (Balsiger et al. 1986). This is because the photoprocessing of interstellar ices leads to a $\mathrm{C}: \mathrm{O}$ ratio in the dust which is at least 4 times the solar ratio, as pointed out in $\S$ II.

2. The presence of $0.1 \mu \mathrm{m}$ particles as basic constituents of the aggregates. - The size of the core-mantle grains within the aggregates determines the highest temperature achievable for high values of the porosity. As can be seen in Figure 8 , the temperature of individual particles peaks in the $0.1 \mu \mathrm{m}$ range. Very small or significantly larger particles are cooler than required to reproduce the observed excess emissions. For example, if we were to use particles with a size of $0.5 \mu \mathrm{m}$ as inclusions in the aggregates, the porosity needed to fit the observations would be similar to those given in the fourth row in Table 2. Furthermore, as demonstrated by Greenberg, Zhao, and Hage (1989), the ratio of the 3.4 to $9.7 \mu$ m excess emissions is critically dependent on the dust temperature. Therefore, the degree of consistency found between the porosities needed to fit both emissions would be lost if substantially lower aggregate temperatures were used.

We should mention here that in modeling a mean interstellar grain silicate core size we were originally influenced by the fact that the size distribution given by McDonnell et al. in 1987 presented insuperable or at least great difficulties (i.e., requiring too high porosities) in reproducing the observed spectral excesses unless the basic grain temperature was significantly higher than that using a canonical (Greenberg 1985) core radius of $0.05 \mu \mathrm{m}$. Since $0.1 \mu \mathrm{m}$ radius silicate cores (the radius at which the temperature peaks; see Fig. 8) are too large because of cosmic abundance constraints (Greenberg 1978), we adopted an intermediate compromise core radius of $0.075 \mu \mathrm{m}$ as the maximum possibility. If we were to use a radius of 0.05 $\mu \mathrm{m}$ in conjunction with the revised Giotto size distribution, the required porosities would be somewhat higher than shown in Table 2, but not impossibly so.

\section{b) Predictions}

We have shown that the amount of excess emission at 9.7 and $3.4 \mu \mathrm{m}$ depends critically on the size spectrum and temperature of the coma dust. Since the size spectrum of the dust is likely to be very variable, it is difficult to predict the behavior of the excess emission as a function of, for example, the cometSun distance. However, we do envisage the following scenario:

At the moment the coma dust aggregates are lifted from the comet surface, they may still contain the ice material. We would expect that fragmentation of such aggregates is inhibited by the cohesive force of the ices. As the ices evaporate, fragmentation should occur more rapidly. For comets which are well beyond, say, $1 \mathrm{AU}$ from the Sun, ice evaporation is 
relatively slow and therefore the relative number of small particles in the size distribution should be less than for comets within $1 \mathrm{AU}$, where the evaporation rate is higher. Thus larger particles are more dominant in the size distribution at larger solar distances. Since the large particles are cooler and have a low mass absorption coefficient compared with small particles, there should be substantially less excess emission at 9.7 and 3.4 $\mu \mathrm{m}$. Furthermore, we expect that if the $9.7 \mu \mathrm{m}$ feature were present it would have to be broad, because the particles are neither small nor porous enough to give the narrow emission seen at $\approx 1 \mathrm{AU}$ or closer to the Sun. We note that the lack of a 9.7 $\mu \mathrm{m}$ emission feature for solar distances substantially greater than $1 \mathrm{AU}$ has been reported by Gehrz and Ney (1986). The lack of small particles alone, even without a decrease in temperature, can account for the absence of the $9.7 \mu \mathrm{m}$ emission (see Fig. 5). This is consistent with the fact that the cometary sunward spike which is known to consist of large grains does not exhibit a $9.7 \mu \mathrm{m}$ emission (Ney 1988). For solar distances substantially less than $1 \mathrm{AU}$ there should be a higher degree of fragmentation and consequently stronger 9.7 and 3.4 $\mu \mathrm{m}$ emissions. The ratio $F^{\mathrm{ex}}(9.7) / F^{\mathrm{ex}}(3.4)$ could be lower because the $3.4 \mu \mathrm{m}$ excess emission is more strongly affected by the temperature rise which is due to the decreased comet-Sun distance. However, too close to the Sun we should also anticipate a significant evaporation of the organic refractory material which produces the $3.4 \mu \mathrm{m}$ emission. Quantitative prediction of how the $3.4 \mu \mathrm{m}$ feature rises and falls as the comet approaches the Sun depends on the relative volatility and abundances of the different compounds in the organic refractory material, which are not certain. However, qualitatively one would predict a maximum in the $3.4 \mu \mathrm{m}$ emission and a subsequent drop as the comet approaches to well within $1 \mathrm{AU}$.

\section{c) Spatially Extended Molecular Emission}

Although the major emphasis here has been on the solid component of the comet coma, it has to be remarked that the chemical and morphological structure of porous aggregates of core-mantle interstellar dust leads naturally to the contribution of some of the gas components. For example, Eberhardt $e t$ al. (1987) argued that a part of the overabundance of CO as well as its spatial distribution could be attributed to organics shed from the dust. The high-temperature dust leads to evaporation of the less refractory organic mantle components, which, when exposed to solar ultraviolet radiation, may be photodissociated into smaller molecular components. From laboratory studies it has been established that interstellar dust should contain CN groups (Grim and Greenberg 1987b; Agarwal et al. 1985) and that CO groups in carboxylic acid fragments (Agarwal et al. 1985; Schutte and Greenberg 1986) are a natural consequence of ultraviolet processing of interstellar ices. The presence of CN jets (A'Hearn et al. 1986) and $\mathrm{CO}$ as well as possibly $\mathrm{C}_{3} \mathrm{H}^{+}$(Marconi et al. 1989) as distributed species in the coma associated with the dust is a natural consequence, but these amounts would be exceedingly small were it not for the large surface area and higher temperatures provided by the porous dust. If one restricted the source of the CO only to compact small particles, the "submicrometer particles" in the language of A'Hearn et al., the number and mass of such particles in the given mass distribution up to $10^{-11} \mathrm{~g}(\approx 1 \mu \mathrm{m})$ would be totally inadequate. This is readily demonstrated by the following argument. Let us estimate the amount of $\mathrm{CO}$ coming from the dust to be given by $M(\mathrm{CO}) /$ $M($ gas $) \approx 0.03$, based on the results of Eberhardt et al. (1987), i.e., about half of the total $\mathrm{CO}$ production. Using $M($ gas $)=2.55 \times 10^{7} \mathrm{~g} \mathrm{~s}^{-1}$, we get $M(\mathrm{CO}) \approx 8 \times 10^{5} \mathrm{~g} \mathrm{~s}^{-1}$. The dust production rates for $M<10^{-11} \mathrm{~g}$ are, for the Giotto, $V e g a 1$, and Vega 2 size distributions, $M_{d}^{G}\left(<10^{-11}\right)=4 \times 10^{5}$ $\mathrm{g} \mathrm{s}^{-1}, M_{d}^{V 1}\left(<10^{-11}\right)=4 \times 10^{5} \mathrm{~g} \mathrm{~s}^{-1}$, and $M_{d}^{V 2}\left(<10^{-11}\right)=$ $2 \times 10^{5} \mathrm{~g} \mathrm{~s}^{-1}$. Thus the ratio of the $\mathrm{CO}$ production rate to the submicron particle production rate is $M(\mathrm{CO}) / M_{d}>2$. This is an impossible condition, implying that the mass of the daughter molecule is greater than the mass of the parent molecules.

How can we then, as we must, attribute any significant amount of $\mathrm{CO}$ to the dust? The answer must be that the $\mathrm{CO}$ comes from higher mass particles as well. But if these larger particles were solid, not only would their temperatures be too low to evaporate the organics, but they would also contribute too small a surface area for evaporation. Both of these objections are resolved by the fact that the coma dust is highly porous and consists of submicron particles. If we extend the possible source of the $\mathrm{CO}$ to particles up to a mass corresponding to, say, a limiting mass of about $10^{-8} \mathrm{~g}$, we would arrive at the corresponding results $M(C O) / M_{d}^{G}\left(<10^{-8}\right)=0.3$, $M(\mathrm{CO}) / M_{d}^{V 1}\left(<10^{-8}\right)=0.04$, and $M(\mathrm{CO}) / M_{d}^{V^{2}}\left(<10^{-8}\right)=0.1$, which are all at least less than unity. When consideration of fragmentation far out in the coma is included to produce more small particles in the size spectrum than appear closer in, we see how, indeed, fluffy particles are capable of providing a source of the CO. Similar arguments apply to the other molecules which are presumed to come from the dust because they are seen so far out that no other parent "molecule" than the dust seems reasonable from the point of view of photodissociation lifetimes.

\section{d) Interplanetary Dust}

The concept of porous aggregates of interstellar dust may be extended toward explaining some key characteristics of chondritic porous interplanetary dust particles (IDPs; Hage and Greenberg $1990 \mathrm{~b}$ ). This was already partly recognized by Fechtig (1984), who looked for an explanation of the fact that the Pioneer 10 and Pioneer 11 dust penetration experiments measured a constant flux out to $20 \mathrm{AU}$, while the optical experiment observed a decrease of the dust number densities out to 3.3 AU until no scattered light was recorded farther out. In addition, the interplanetary dust temperature dependence on the distance to the Sun is $r^{-\mathbf{0 . 3 3}}$ (Dumont and LevasseurRegourd 1988), unlike the expected behavior of $r^{-0.5}$, i.e., like a blackbody. These phenomena could be understood by combining our present work with the theory of Mukai and Fechtig (1983), in which the heating of porous particles consisting of small core-mantle units leads to slowly increasing density as the particles circulate about the Sun. Those which remain farther out for a greater fraction of time are less processed in this way and therefore more porous than those which come closer to the Sun. The increase of porosity could partly counteract the decrease in dust temperature due to the increase in distance to the Sun and thus reproduce what is observed. The more distant and more porous dust particles would also have a lower albedo (Hage and Greenberg 1990a), possibly low enough to explain the Pioneer results. In addition, this could provide a basis to explain the anomalously high ratio of infrared emission to visual scattering observed for the zodiacal light particles beyond 1 AU (see, e.g., Hong and Um 1987).

The IDPs collected in the Earth's atmosphere may also be conjectured to have started out as coma dust particles, i.e., porous aggregates of interstellar dust, which have traveled for 
some time in the solar system. Although the chondritic porous IDPs show no evidence of a bird's nest structure as shown in Figure $2 a$, they do appear as aggregates of more or less spherical silicate particles of about $0.1 \mu \mathrm{m}$ size which are like the pieces of the silicate cores in the interstellar dust. We note that when the interstellar dust grains (pictured in Fig. 1b), as units in porous aggregates, lose a portion of their mantles and are compacted, the remaining silicate core segments, which are hidden under the mantles in the bird's nest model, begin to appear like the silicates in the IDPs (see Fig. $2 b$ ). Furthermore, although the organic mantles are not "seen" in the IDP electron micrographs, their presence is immediately detected when the IDP sample is examined with Raman spectroscopy (Wopenka 1988). In fact, the silicate signature which dominates the normal infrared spectra disappears because the visible radiation cannot penetrate to it through an absorbing organic mantle. The fact that the mean silicate particle size in IDPs is like that of the interstellar core pieces, and each silicate has an organic refractory coating, is certainly suggestive of the interstellar origin. The bird's nest morphological structure could be lost because of the removal of a large part of the organic mantles in the originating coma dust aggregates. This would appear to answer Walker's (1988) objection to the connection between interstellar dust and the IDPs via evolution of bird's nest comet debris. The precise amount of organics in the IDPs is not clear (Wopenka 1988), but it is undoubtedly much less than in coma dust. If one assumes an organic mantle thickness of about $50 \times 10^{-10} \mathrm{~m}$ (about $20 \%$ by volume, or $10 \%$ of the mass) then the mean density of fully compact IDP material would be $3.2 \mathrm{~g} \mathrm{~cm}^{-3}$. It is often stated that IDPs have a low density in the range of $1 \mathrm{~g} \mathrm{~cm}^{-3}$. This would imply a porosity of about 0.7 , which is, as expected, substantially lower (and consequently gives a higher albedo) than that of the coma dust. As already demonstrated, $P=0.7$ is too low to be acceptable for coma dust, and furthermore, with such a small fraction of organics, particles like the IDPs would be totally unable to produce the required high temperature. Therefore, although one may argue that IDPs evolve from comet fragments, they cannot be used directly to provide a theory of coma dust. In fact, they are futher removed from coma dust than coma dust is from interstellar dust, and this is reasonable in view of the fact that they are subjected to a much higher degree of physical processing, during their typically $10,000 \mathrm{yr}$ interplanetary lifetime, than the comet nucleus material is exposed to. It seems appropriate at this point to present a recent quote from Brownlee (1990), who has made a study of the composition of comet Halley dust particles and their relationship to IDPs ("Brownlee particles") and to the "Greenberg model." After pointing out various degrees of consistency and inconsistency, he says, "A more convincing connection between the data and the [Greenberg] model would be evidence for radiation processing of the organic component or evidence that the organic material actually occurs as thick mantles over silicate cores." The core-mantle structure has been amply shown here to be an absolutely essential feature of the basic units in the coma dust aggregates. The characteristic evolutionary trend as seen in the interplanetary particle distribution from the point of view of Pioneer 10/11 data has been summarized by Fechtig (1984): "From this picture it would seem that the dust experiments on Pioneer 10/11 see first a mixture of Brownlee particles (high albedos) and Greenberg particles (lower albedos) and in the outer solar system only the Greenberg particles are left."
VIII. CONCLUSIONS

We have provided quantitative evidence to show that, in order to satisfy simultaneously such independent properties of coma dust as (1) its $3.4 \mu \mathrm{m}$ emission, (2) its $9.7 \mu \mathrm{m}$ emission (amount and shape), (3) its mass distribution, and (4) its mass spectroscopic composition, the comet nucleus is most likely a low-density aggregate of interstellar dust. The basic units of the coma dust are the submicron silicate core-organic refractory mantle particles characteristic of diffuse cloud interstellar dust which are aggregated into very porous structures. These coremantle unit particles have lost their original ices and the more volatile part of their organic refractory material after leaving the comet nucleus. The comet nucleus porosity deduced from the coma dust porosity is then most likely in the range $0.6<$ $P_{\text {nuc }}<0.83$, which leads to a comet density of $0.6 \mathrm{~g} \mathrm{~cm}^{-3}>$ $\rho_{\text {nuc }}>0.26 \mathrm{~g} \mathrm{~cm}^{-3}$. These values are consistent with comet nucleus densities derived by others and observations of lowdensity meteors. Next to the derived density and porosity of the comet nucleus, the most important property is that it must be made of submicron core-mantle particles. The morphological characteristics of core-mantle units and fluffy aggregates lead to the notion that comets formed by relatively gentle aggregation of protosolar nebula interstellar dust and that the nucleus of a comet as it exists today has preserved both of these properties to a very high order. Comet nuclei must therefore represent very closely the material out of which the solar system was born. Only if the comet nucleus material can be retrieved from its depths, and maintained intact cryogenically for laboratory studies, may we hope to study not only its atomic and molecular compositions but also its morphology. Microprobes are being developed (Bradley and Brownlee 1986) which will make such investigations of submicron structures possible. On the basis of the interstellar dust model, individual grains whose mean lifetime before becoming part of a comet is about $5 \times 10^{9} \mathrm{yr}$ will reveal cosmochemical evolution not only of the solar system but dating back a further 5 billion years before the Earth's beginning - back to the earliest stages of the chemical evolution of the Milky Way. Dramatic differences in isotopic abundances could be expected on scales of microns. The next twenty to thirty years should be exciting ones indeed for studies of our origins. Until the Rosetta Comet Nucleus Sample Return Mission arrives with, one hopes, an intact piece of comet material that will either confirm or negate our results, we feel as confident as Hercule Poirot when he said, "Although I have now arrived at what I believe to be the true solution of the case, I have no material proof of it. I know it is so, because it must be so, because in no other way can every single fact fit into its ordered and recognized place" (Christie 1935).

We would like to acknowledge partial support for this research from NASA grant NGR33018148. We thank R. T. Wang for providing us with a fast and reliable algorithm to calculate the light scattering by spheres. We thank J. A. M. McDonnell and members of the Unit for Space Sciences, University of Kent, for assistance when this work was in progress. We are grateful to Cor van de Mee for carefully reading the manuscript and to the referee for making some useful suggestions. 


\section{REFERENCES}

Agarwal, V. K., Schutte, W. Greenberg J. M. Ferris, J. P. Briggs, R Connor, S., Van de Bult, C. P. E. M., and Baas, F. 1985, Origins of Life, 16, 21 .

A'Hearn, M. F., Feldman, P. D., and Schleicher, D. G. 1983, Ap. J. (Letters), 274, L99.

A'Hearn, M. F. Hoban, S., Brich, P. V., Bowers, C., Martin, R., and Klinglesmith, D. A. III. 1986, Nature, 324, 649 .

Balsiger, H., et al. 1986, Nature, 321, 330.

Bradley, J. R., and Brownlee, D. E. 1986, Science, 231, 1542.

Brownlee, D. E. 1978, in Cosmic Dust, ed. J. A. M. McDonnell (New York: Wiley), p. 295.

1990, in Comet Halley 1986-Worldwide Investigations, Results and Interpretations, ed. J. Mason and P. Moore (Chichester: Ellis Horwood), in press.

Chlewicki, G. 1986, Ph.D. thesis, Leiden University.

Chlewicki, G., and Greenberg, J. M. 1990, Ap. J., in press.

Christie, A. 1935, Murder in Mesopotamia (copyright 1935-1936, A. Christie).

Danks, A. C., Encrenaz, T., Bouchet, P., LeBertre, T., and Chalabaev, A. 1987, Astr. Ap., 184, 329 .

Day, K. L. 1979, Ap. J., 234, 158

Draine, B. T. 1985, Ap. J. Suppl., 57, 587.

Draine, B. T., and Lee, H. M. 1984, Ap. J., 285, 89.

Dumont, R., and Levasseur-Regourd, A.-C. 1988, Astr. Ap., 191, 154.

Eberhardt, P., et al. 1987, Astr. Ap., 187, 481.

Fechtig, H. 1984, Adv. Space Res., 4(No. 9), 5.

Feldman, P. D., et al. 1987, Astr. Ap., 187, 325.

Gehrz, R. D., and Ney, E. P. 1986, in Exploration of Halley's Comet (ESA SP-250), p. 1010.

Greenberg, J. M. 1971, Astr. Ap., 12, 204

. 1977, in Comets, Asteroids, Meteorites, ed. A. H. Delsemme (Toledo: University of Toledo), p. 491.

p. 187 .

Press) p. 131

1985, Phys. Scripta, 11, 14

1986a, Nature, 321, 385.

1986b, in Asteroids, Comets and Meteors II, ed. C. I. Lagerkvist, B. A Lindblad, H. Lundstedt, and H. Rickman (Uppsala: Uppsala University Press), p. 221.

p. 177 .

1986c, in Light on Dark Matter, ed. F. P. Israel (Dordrecht: Reidel),

1988, in Dust in the Universe, ed. M. Bailey and D. A. Williams (Cambridge: Cambridge University Press), p. 121.

Greenberg, J. M., and Gustafson, B. 1981, Astr. Ap., 93, 35.

Greenberg, J. M., Zhao, N. S., and Hage, J. I. 1989, Adv. Space Res., 9 (No. 3 ), 3.
Grim, R. J. A., and Greenberg, J. M. 1987a, Astr. Ap., 181, 155.

$1987 b$, Ap. J. (Letters), 321, L91.

Hage, J. I., and Greenberg, J. M. 1990a, 361, 251. $1990 b$, in preparation.

Hanner, M. S., Tokunaga, A. T., Golisch, W. E., Gripe, D. M., and Kaminski, C. D. 1987 , Astr. Ap., 187, 653 .

Hong, S. S., and Greenberg, J. M. 1978, Astr. Ap., 69, 341

Hong, S. S., and Um, I. K. 1987, Ap. J., 320, 928.

Jessberger, E. K. 1989, in Proc. Varenna Enrico Fermi School, Solid State Astrophysics, in preparation.

Kissel, J., and Krueger, F. R. 1987, Nature, 326, 755

Krankowsky, D., et al. 1986, Nature, 321, 326.

Larson, H. P., Weaver, H. A., Mumma, M. J., and Drapatz, S. 1989, Ap. J., 338, 1106.

Leger, A., d'Hendecourt, L., and Boccara, N., eds. 1987, Polycyclic Aromatic Hydrocarbons and Astrophysics (Dordrecht: Reidel).

Marconi, M. L., Korth, A., Mendis, D. A., Lin, R. P., Mitchell, D. L., Réme, H., and d'Uston, C. 1989, Ap. J. (Letters), 343, L77.

Maxwell-Garnett, J. C. 1904, Phil. Trans. R. Soc. London, A, 203, 835.

Mazets, E. P., et al. 1987, Astr. Ap., 187, 699

McDonnell, J. A. M., et al. 1987, Astr. Ap., 187, 719

McDonnell, J. A. M., Pankiewicz, G. S., Birchley, P. N. W., Green, S. F., and Perry, C. H. 1989, in Proc. Workshop on Analysis of Returned Comet Nucleus Samples (Milpitas, California), in press.

Mukai, T., and Fechtig, H. 1983, Planet. Space Sci., 31, 655.

Ney, E. P. 1988, private communication.

Olsson-Steel, D. 1989, in Highlights Astr., 8, 313.

Rickman, H., Kamel, L., Festou, M., and Froeschle, C. 1988, in Symposium on the Diversity and Similarity of Comets (ESA SP-278), p. 471.

Roche, P. F., and Aitken, D. K. 1984, M.N.R.A.S., 408, 481.

Ruzmaikina, T. V., and Maeva, S. V. 1988, paper presented at COSPAR Conference (Helsinki).

Sagdeev, R. Z., Elyasberg, P. E., and Moroz, V. I. 1988, Nature, 331, 240.

Schutte, W. 1988, Ph.D. thesis, Leiden University.

Schutte, W., and Greenberg, J. M. 1986, in Light on Dark Matter, ed. F. P. Israel (Dordrecht: Reidel), p. 229.

Sekanina, Z., and Yeomans, D. K. 1985, A.J., 90, 2331

Smirnov, V. N., Vaisberg, O. L., and Anasimov, S. 1987, Astr. Ap., 187, 774. van de Hulst, H. C. 1957, Light Scattering by Small Particles (New York: Wiley; reprinted 1981, New York: Dover).

Verniani, F. 1969, Space Sci. Rev., 10, 230.

.1973, J. Geophys. Res., 78, 8429.

Walker, R. M. 1988, in Infrared Observations of Comets Halley and Wilson and Properties of the Grains, ed. M. S. Hanner (NASA CP-3004), p. 53.

Wopenka, B. 1988, Earth Planet. Sci. Letters, 88, 221.

J. M. GreEnBerg and J. I. HAGE: Laboratory Astrophysics, University of Leiden, Postbus 9504, 2300 RA Leiden, The Netherlands 
PLATE 1

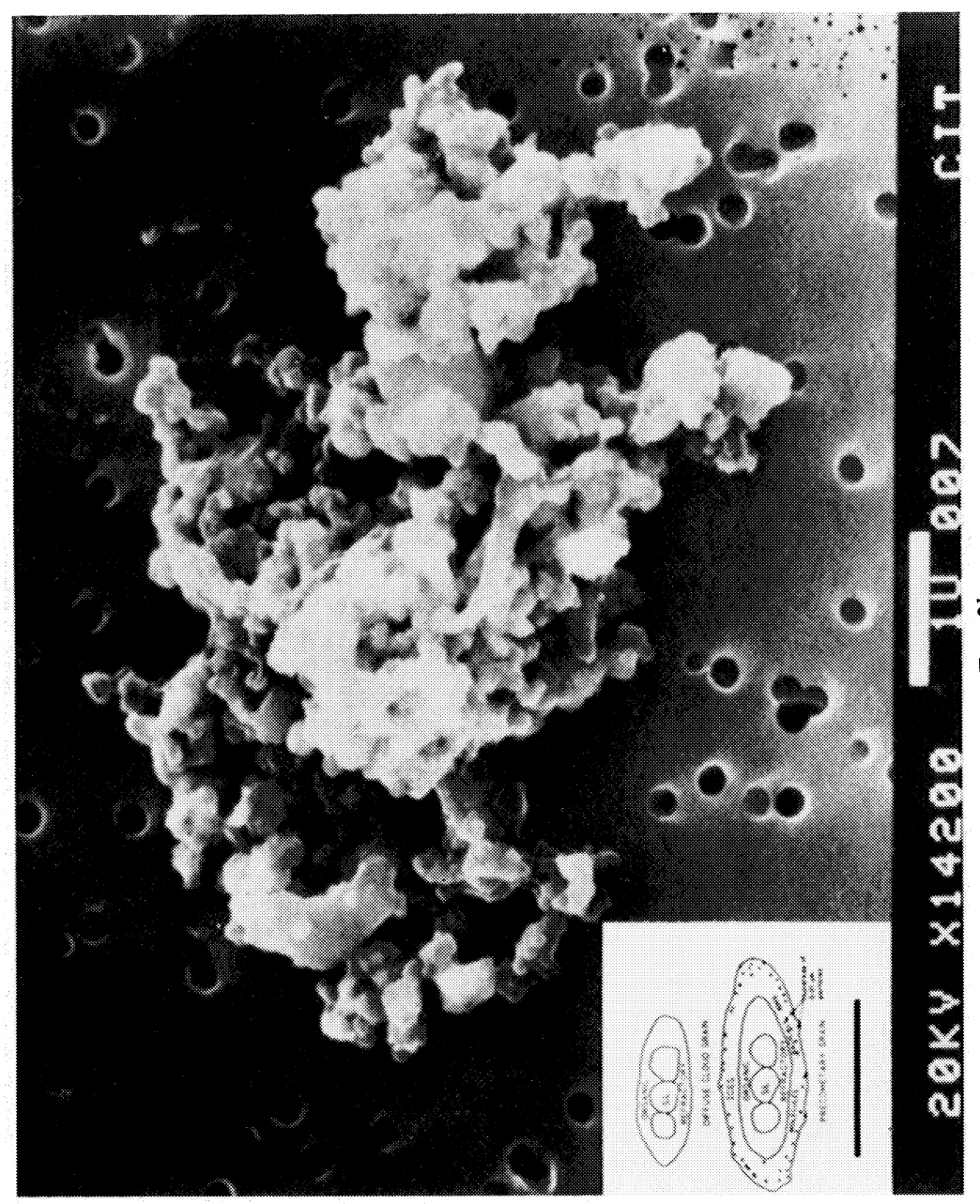

总总

变

过

ㅎํ

음

는

总

.

总溇

实

तิ ธิธง

ن

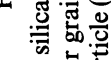

氜

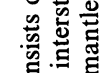

ธำ

둥ㅇㅇㅇ

항

응요

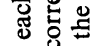

은

E्ञ

营焉

卷

焉

品 范

政

要

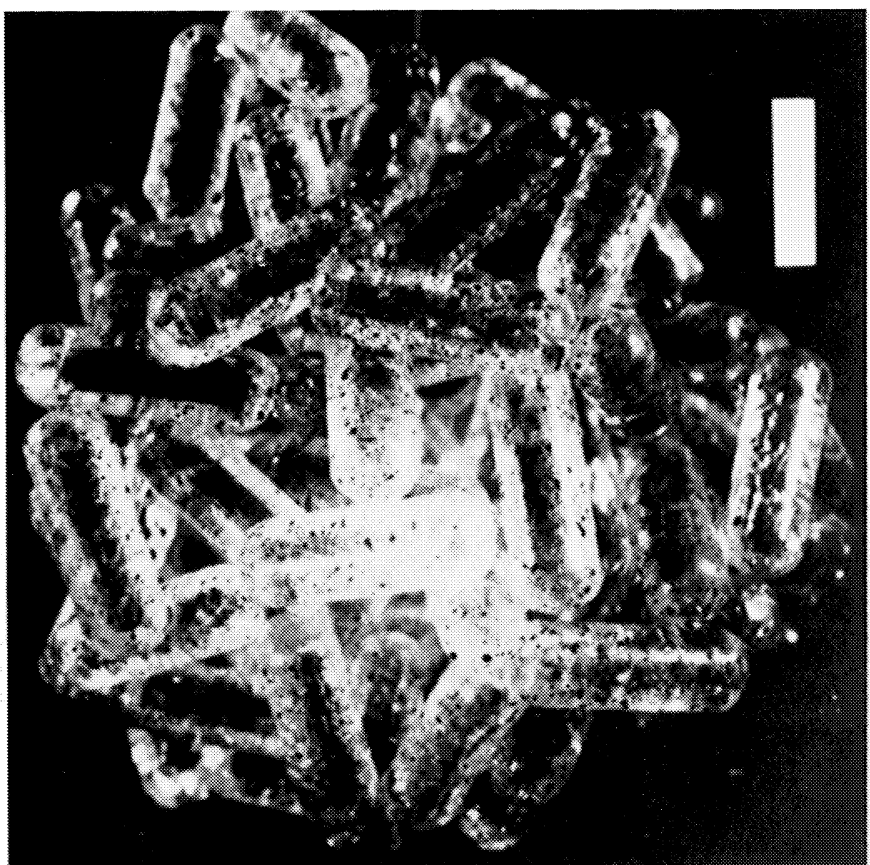

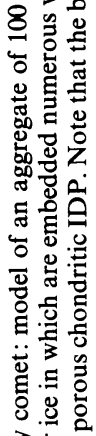

त 焉空

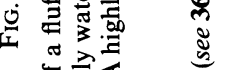

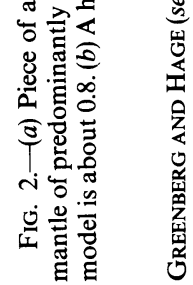

(C) American Astronomical Society - Provided by the NASA Astrophysics Data System 